\title{
Screening and Identification of Differentially Expressed Genes Expressed among Left and Right Colon Adenocarcinoma
}

\author{
Jing Han, ${ }^{1}$ Xue Zhang, ${ }^{1}$ Yang Yang, ${ }^{2}$ Li Feng, ${ }^{1}$ Gui-Ying Wang $\mathbb{D}^{2},{ }^{2}$ and Nan Zhang ${ }^{3}$ \\ ${ }^{1}$ Department of Medical Oncology, The Fourth Hospital of Hebei Medical University, Shijiazhuang, Hebei 050000, China \\ ${ }^{2}$ Second Department of Surgery, The Fourth Hospital of Hebei Medical University, Shijiazhuang, Hebei 050000, China \\ ${ }^{3}$ Department of Thoracic Surgery, The Fourth Hospital of Hebei Medical University, Shijiazhuang, Hebei 050000, China
}

Correspondence should be addressed to Gui-Ying Wang; wangguiyinghb11@163.com

Received 1 September 2019; Revised 6 November 2019; Accepted 17 December 2019; Published 21 January 2020

Academic Editor: Kazim Husain

Copyright () 2020 Jing Han et al. This is an open access article distributed under the Creative Commons Attribution License, which permits unrestricted use, distribution, and reproduction in any medium, provided the original work is properly cited.

\begin{abstract}
Purpose. Colon adenocarcinoma (COAD) is the third most common malignancy globally and is further categorized as left colon adenocarcinoma (LCOAD) or right colon adenocarcinoma (RCOAD) depending on the location of the primary tumor. The therapeutic outcome and long-term prognosis for patients with COAD are less than satisfactory, and this may be associated with tumor location. Therefore, it is important to investigate the genetic differences in COAD at different sites. Patients and Methods. Public data associated with COAD were downloaded from the Gene Expression Omnibus (GEO) database. Differentially expressed genes (DEGs) were identified using R software (version 3.5.3), and functional annotation of DEGs was performed using Gene Ontology (GO) and Kyoto Encyclopedia of Genes and Genomes (KEGG) analyses. A protein-protein interaction network was constructed, hub genes were identified and analyzed, and data mining using Gene Expression Profiling Interactive Analysis (GEPIA) was conducted. Results. A total of 286 DEGs were identified between LCOAD and RCOAD. Additionally, 10 hub genes associated with COAD at different locations were screened, namely, CDKN2A, IGF1R, MDM2, SMAD3, SLC2A1, GRM5, PLCB4, FGFR1, UBE2V2, and TNFRSF10B. The expression of cyclin-dependent kinase inhibitor 2A (CDKN2A) and solute carrier family 2 member 1 (SLC2A1) was significantly associated with pathological stage $(P<0.05)$. COAD patients with high expression levels of CDKN2A exhibited poorer overall survival (OS) times than those with low expression levels $(P<0.05)$. Conclusion. CDKN2A expression was significantly different between LCOAD and RCOAD and was closely related to the prognosis of COAD. It is of great value for further understanding of the pathogenesis of LCOAD and RCOAD.
\end{abstract}

\section{Introduction}

Colon adenocarcinoma (COAD) is the third most common malignancy worldwide, accounting for $10.0 \%$ of all new cancer cases, and is one of the leading causes of cancerassociated mortality [1]. The incidence of COAD has increased year on year and is closely associated with genetic, environmental, and dietary changes, as well as colonic mucosal hyperplasia and the canceration of adenomatous polyps [2]. With the development of targeted therapy, great progress has been made in the treatment of COAD, but the therapeutic outcome and long-term prognosis of patients remain unsatisfactory. It has been suggested that this may be associated with the location of the tumor; thus, the investigation of differences in the incidence of COAD at different sites is particularly important.

Based on tumor location, COAD includes at least two types [3], left colon adenocarcinoma (LCOAD) and right colon adenocarcinoma (RCOAD). LCOAD refers to tumors from the splenic flexure of the colon to the sigmoid colon, and RCOAD refers to tumors between the ileocecal region and the transverse colon [4]. In addition to their different origins, LCOAD and RCOAD also have different clinical manifestations, histological types, molecular characteristics, prognoses, modes of metastasis, and treatment options [3], which are reflected in the following aspects.

In terms of clinical manifestation, hematochezia and changes in bowel habits are more frequently associated 
with LCOAD, while iron-deficiency anemia caused by occult blood loss is more common in patients with RCOAD [5]. The data showed that RCOAD patients were more likely to be female, of older age, with larger tumor diameters, poor differentiation, later Tumor-Node-Metastasis stages, and shorter survival times compared with LCOAD patients $[6,7]$. In the past 30 years, the incidence of RCOAD has risen, and its incidence is now reportedly higher than that of LCOAD [8]. From a molecular perspective, RCOAD and LCOAD are two separate entities. The fundamental reason for the obvious difference between RCOAD and LCOAD lies in the difference of molecular typing. For example, in the RCOAD, there are high mutations of genes, methylation, BRAF (B-Raf Proto-Oncogene, Serine/Threonine Kinase) mutation, serrated pathway, and inflammatory. And the prognosis of the RCOAD is poor [9]. However, in the LCOAD, there exist chromosomal instability, amplification of EGFR1 (Epidermal Growth Factor Receptor 1) and EGFR2 (Epidermal Growth Factor Receptor 2), EGF (Epidermal Growth Factor) signal transduction, and Wnt signal transduction. $13 \%$ of the LROAD with BRAF mutation has a poor prognosis, while $87 \%$ without BRAF mutation will have a good prognosis [9]. RCOAD is related to KRas and Serine/ threonine-protein kinase B-raf (BRAF) mutations of defect mismatch repair genes and microRNA-31, while LCOAD is closely associated with chromosome instability, p53, NRas, and microRNA-146a, microRNA-147b, and microRNA1288 [10]. However, Gao et al. [11] showed no significant difference in the expression levels of MLH1, MSH2, MSH6, PMS2, $\beta$-tubulin III, p53, Ki67, topoisomerase Ii $\alpha$, and BRAF gene mutations between the two types of COAD. A number of studies have reported significant differences in p53 gene mutation and protein expression between RCOAD and LCOAD [12-14], while another study has shown no significant correlation between p53 protein expression and tumor location [15]. Therefore, it is significantly necessary to identify the differentially expressed genes between RCOAD and LCOAD.

Bioinformatics is a comprehensive field that integrates biology, computer science, and mathematics [16]. With the development of sequencing technology, bioinformatics data has rapidly accumulated and is widely used in medicine and drug development. Concurrently, much gene expression profile data have been generated [17], and efficient data mining has become a bioinformatics research hotspot. The development of bioinformatics also provided a novel approach for the discovery and identification of differentially expressed genes (DEGs) between LCOAD and RCOAD [18].

In the present study, COAD gene chip data from the Gene Expression Omnibus (GEO) were analyzed to identify DEGs and hub genes between LCOAD and RCOAD, construct an interaction network of DEGs, and conduct Gene Ontology (GO) and Kyoto Encyclopedia of Genes and Genomes (KEGG) analyses between these genes. These DEGs and hub genes may provide new ideas to study the differences between LCOAD and RCOAD and the subsequent development of targeted therapy.

\section{Materials and Methods}

2.1. Access to Public Data. The GEO (http://www.ncbi.nlm. nih.gov/geo) is an open-source platform for the storage of genetic data [19]. Two expression profiling datasets (GSE81558 (GPL15207 platform) and GSE75317 (GPL570 platform)) were, respectively, downloaded from the GEO database. The GSE81558 dataset includes 9 normal colorectal tissues, 19 liver tissues from colorectal liver metastasis patients, 12 rectum tissues from primary colorectal tumor patients, 9 left colon tissues from primary colorectal tumor patients, and 2 right colon tissues from primary colorectal tumor patients. This study mainly aimed to identify the differentially expressed genes between left colorectal tumors and right colorectal tumors. Therefore, we chose only 9 LCOAD and 2 RCOAD samples from the GSE81558 dataset based on the source type. Similarly, 33 LCOAD samples and 26 RCOAD samples were selected from GSE75315 (GPL570 platform).

2.2. DEGs Identified Using R Software. R software (version 3.5.3) is used to distinguish DEGs between LCOAD and RCOAD tissue samples. If one probe set does not contain the homologous gene, or if one gene has numerous probe sets, the data is removed. $P<0.05$ is considered to indicate a statistically significant difference. The DEGs are presented as volcano plots, generated using SangerBox software (http:// sangerbox.com/), and Venn diagrams were constructed using FunRich software (http://www.funrich.org).

\subsection{Functional Annotation of DEGs Using KEGG and GO} Pathway Enrichment Analyses. The Database for Annotation, Visualization, and Integrated Discovery (DAVID) (https://david.ncifcrf.gov/home.jsp; version 6.8) is an online suite of analysis tools with an integrated discovery and annotation function [20]. The GO resource is widely used in bioinformatics and covers three aspects of biology, including biological process (BP), cellular component (CC), and molecular function (MF) [21]. KEGG (https://www.kegg.jp/) is one of the most commonly used biological information databases worldwide [22]. DAVID was used to perform GO and KEGG analyses of DEGs, and $P<0.05$ was considered to indicate a statistically significant difference.

2.4. Construction of a Protein-Protein Interaction (PPI) Network. Search Tool for the Retrieval of Interacting Genes (http://string.embl.de/), an open-source online tool, was used to construct a PPI network of the identified DEGs, and Cytoscape visualization software version 3.6.1 [23] was used to present the network [24]. A confidence score $>0.4$ was considered as the criterion of judgment, which may filter out the critical module.

2.5. Identification and Analysis of Hub Genes. Functional annotation of the genes was performed using KEGG and GO analyses in DAVID. A single coexpression network was constructed using cBioPortal (http://www.cbioportal.org) 
[25]. The Biological Networks Gene Oncology tool (BiNGO) version 3.0.3, one plug-in of the Cytoscape, was used to analyze and visualize the BPs and MFs of each hub gene [26]. OmicShare (http://www.omicshare.com/tools), an open data analysis platform, was subsequently used to perform clustering analysis of these genes.

2.6. Data Mining Using Gene Expression Profiling Interactive Analysis (GEPIA). The correlations between gene expression and pathological stage were ascertained using GEPIA (http://gepia.cancer-pku.cn/), a newly developed interactive web server for analyzing the gene expression data of large consortium projects such as The Cancer Genome Atlas and the Genotype Tissue Expression project [27]. Correlations between pathological stage, overall survival (OS), and the expression of hub genes in COAD were also identified using GEPIA. The correlation between SLC2A1 and GLUT1 expression was tested by GEPIA.

2.7. RT-qPCR Assay. A total of 8 participates were recruited, including 4 LCOAD and 4 RCOAD samples. After surgery, 4 LCOAD samples from LCOAD patients and 4 RCOAD samples from control individuals were obtained. The research conformed to the Declaration of Helsinki and was authorized by the Human Ethics and Research Ethics Committees of the Fourth Hospital of Hebei Medical University. An informed consent was obtained from all participants.

Total RNA was extracted from 4 LCOAD samples and 4 RCOAD samples by the RNAiso Plus (Trizol) kit (Thermofisher, Massachusetts, America) and reverse transcribed to cDNA. RT-qPCR was performed using a Light $\mathrm{Cycler}^{\circledR}$ 4800 System with specific primers for the ten hub genes. Table 1 presents the primer sequences used in the experiments. The RQ values $\left(2^{-\Delta \Delta C t}\right.$, where $C t$ is the threshold cycle) of each sample were calculated and are presented as fold change in gene expression relative to the control group. GAPDH was used as an endogenous control.

2.8. Overall Survival Analysis of the LCOAD and RCOAD. The present study recruited a total of 106 LCOAD and 106 RCOAD patients from the Fourth Hospital of Hebei Medical University. Clinical and histopathological characteristics and follow-up and survival information were available for all patients and were collected retrospectively from medical records. Patients who are aged 30 to 100 years old, are histologically confirmed as colorectal adenocarcinoma [28], do not receive tumor treatment, and have no history of surgery [29] will be screened for inclusion criteria. Exclusion criteria included the following: age $<30$ years old or $>100$ years old, combined with other malignant tumors, operation time more than 1 month after the last examination, and severe heart disease. The expression level of CDKN2A in LCOAD or RCOAD patients was measured by RT-qPCR. In this clinical study, we followed up the patients for 210 months. The endpoint of the study was death from colon adenocarcinoma. This trial and the informed consent forms
TABle 1: Primers and their sequences for PCR analysis.

\begin{tabular}{lc}
\hline Primer & Sequence $\left(5^{\prime}-3^{\prime}\right)$ \\
\hline CDKN2A-hF & ATATAGCTTCAAAAAGCAAAGGC \\
CDKN2A-hR & TTAAAATCAAATCCAGCAACAGG \\
IGF1R-hF & GAAGTTGAGAAGGAATGAAGACA \\
IGF1R-hR & AATCACCCAAGAAAACAAGACAG \\
MDM2-hF & CCAAGGGGGGTAGTAAAGGGTAT \\
MDM2-hR & TAGAAGGCAAGGAAGAAAGGAGT \\
SMAD3-hF & CACTCGGGAATGGGAAAAATGAA \\
SMAD3-hR & AAAAAATAGCCAGGCGTGGTAGC \\
SLC2A1-hF & GCATGGGTGATGTGTGGTTTGAA \\
SLC2A1-hR & AGGGTATCCTCTCCTGGTTTTAG \\
GRM5-hF & AGGACAGTAAACCAGGAAGCAGG \\
GRM5-hR & GAGGTAATTGAATCATAGGGGCG \\
PLCB4-hF & TGCTTTAATTTTATTATACCCCC \\
PLCB4-hR & AAGTCTCAGTCAATCCAGTCCTC \\
FGFR1-hF & GCCAGAGCAAGTGTGGGTTTTAT \\
FGFR1-hR & GATGCGTGTGATTCGGAGAGGGT \\
UBE2V2-hF & AGGTTCACTCCTCATTCTTTTTT \\
UBE2V2-hR & TTTTCCCTATTTGATGTTTCTGT \\
TNFRSF10B-hF & AATATACGCAGGATTTGAAGACG \\
TNFRSF10B-hR & ACATTAAAAAAGGTGAGAAGGGG \\
\hline
\end{tabular}

have been reviewed and approved by the Ethics Review Committee of Fourth Hospital of Hebei Medical University, and the approval number is 2017MEC115. The Kaplan-Meier method was performed to analyze the overall survival. All statistical analyses were conducted using SPSS software (version 21.0), and $P<0.05$ was considered statistically significant.

\section{Results}

3.1. Screening of DEGs between $L C O A D$ and RCOAD. In the GSE81558 dataset, we chose nine LCOAD and two RCOAD samples into this research. And in the GSE75317 dataset, we chose $33 \mathrm{LCOAD}$ and $26 \mathrm{RCOAD}$ samples into this research. Following the analysis of the GSE81558 and GSE75317 datasets, respectively, the differences between LCOAD and RCOAD tissues in GSE81558 and GSE75317 were presented as volcano plots as shown in Figures 1(a) and 1(b), respectively. A Venn diagram revealed 286 common DEGs between the two datasets (Figure 1(c)).

3.2. Functional Annotation for DEGs Using KEGG and GO Analyses. The results of $\mathrm{GO}$ analysis revealed that variations in the BP were predominantly enriched in protein complex assembly, sialylation, oligosaccharide metabolic process, peptidyl-tyrosine, phosphorylation, and apoptotic process. Changes in CC were primarily enriched in intracellular, cellcell junction, peroxisomal matrix, cytosol, and postsynaptic density. Variations in MF were enriched in metal ion binding, sialyltransferase activity, transcription factor activity, sequence-specific DNA binding, nucleic acid binding, and protein binding (Table 2). KEGG analysis demonstrated that DEGs were largely enriched in transcriptional misregulation in cancer, pathways in cancer, and peroxisome (Table 2). 


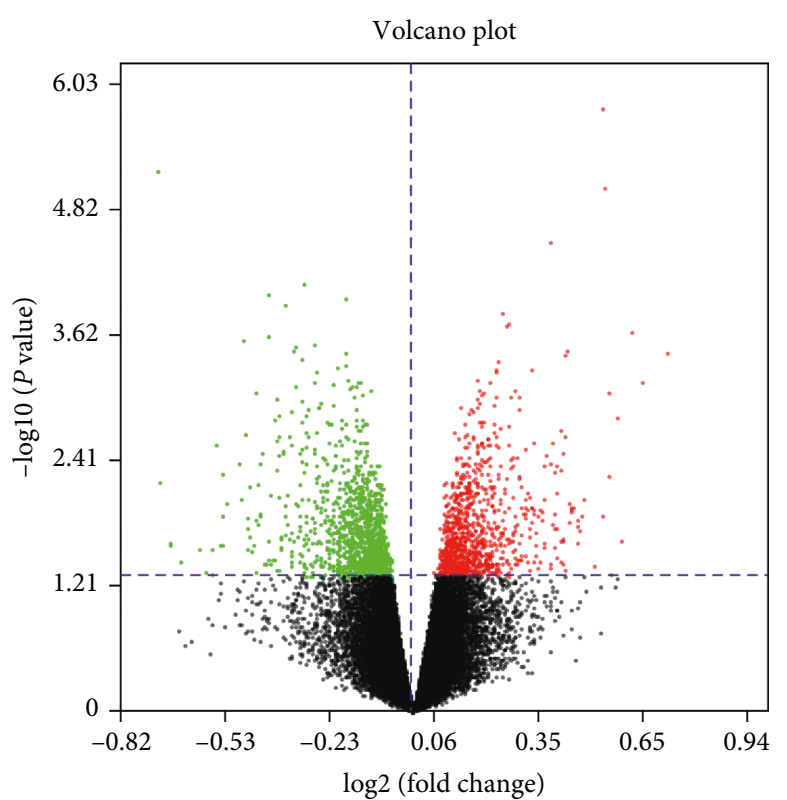

(a)

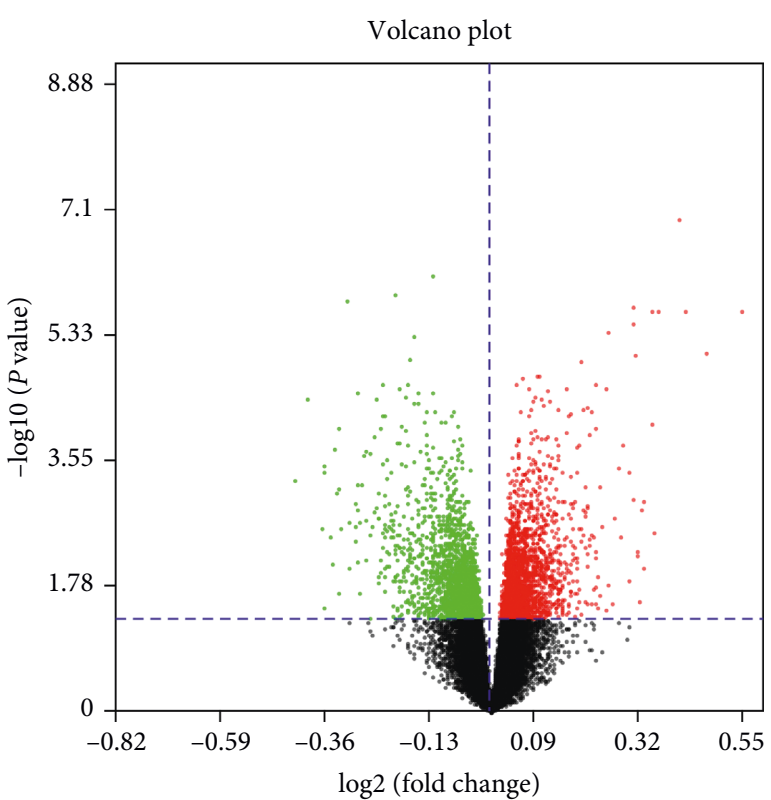

(b)

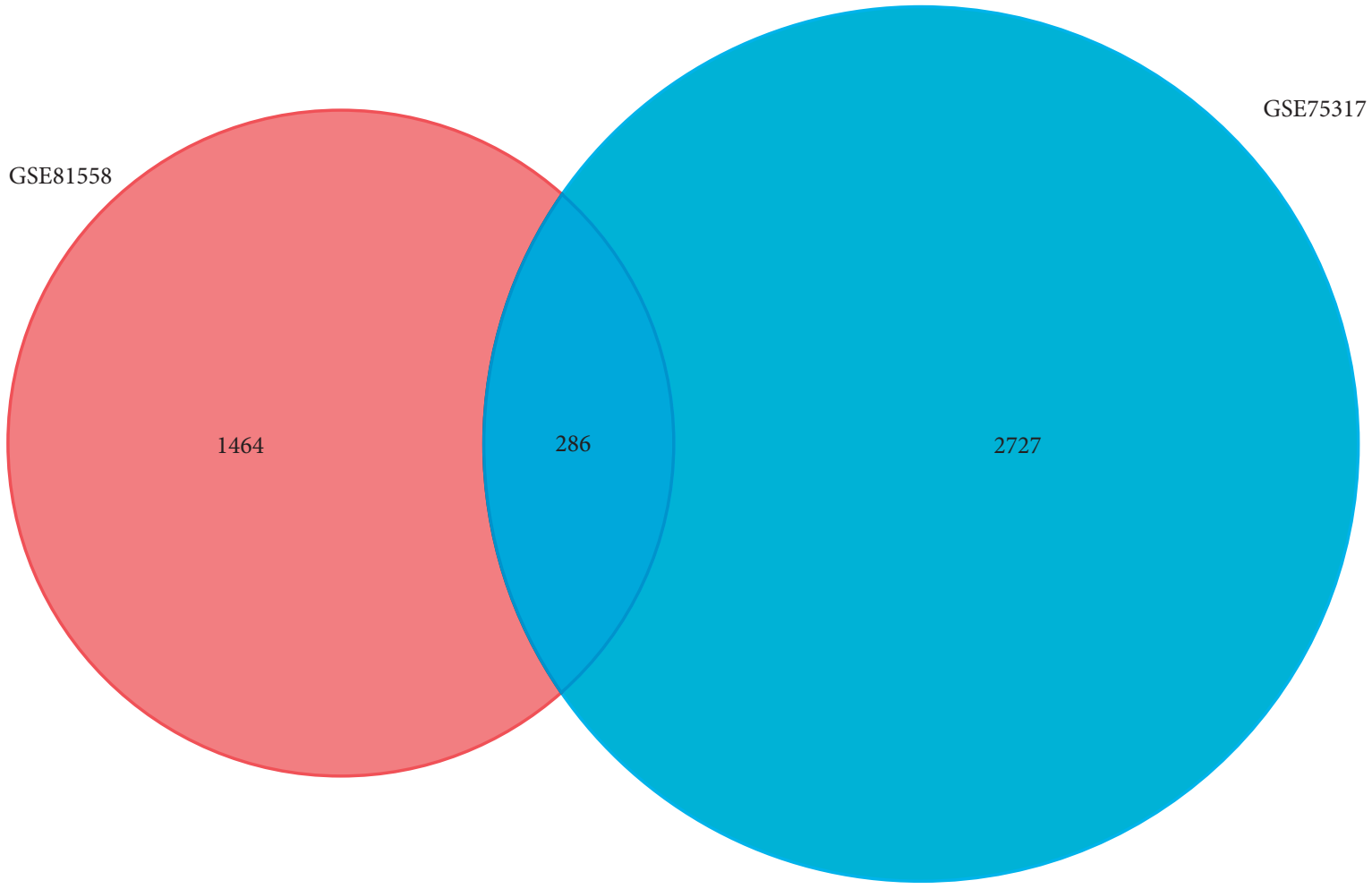

(c)

FIGURE 1: Identification of differentially expressed genes. Volcano plots present the difference between LCOAD and RCOAD samples of the (a) GSE81558 and (b) GSE75317 datasets. (c) Venn diagram identifying 286 common genes between the two datasets.

3.3. Construction of the PPI Network. The construction of a PPI network revealed 264 edges and 159 nodes in the PPI network (PPI enrichment; $P=0.0112$; Figure 2). The network possessed significantly more interactions than expected, highlighting a greater number of interactions between DEGs than expected for a random set of proteins of a similar size from the same genome. Such enrichment indicates that the identified proteins are at least partially associated.

3.4. Hub Gene Selection and Functional Annotation. The following 10 hub genes were identified using Cytoscape, and KEGG and GO analyses were conducted using DAVID: 
TABLE 2: GO and KEGG pathway enrichment analyses of DEGs between left and right COAD.

\begin{tabular}{lccc}
\hline Term & Description & Count in gene set & $P$ value \\
\hline GO:0006461 & Protein complex assembly & 8 & 0.002 \\
GO:0097503 & Sialylation & 4 & 0.003 \\
GO:0009311 & Oligosaccharide metabolic process & 8 & 0.006 \\
GO:0018108 & Peptidyl-tyrosine phosphorylation & 17 & 0.009 \\
GO:0006915 & Apoptotic process & 35 & 9.016 \\
GO:0005622 & Intracellular & 9 & $9.01 E-04$ \\
GO:0005911 & Cell-cell junction & 5 & 0.004 \\
GO:0005782 & Peroxisomal matrix & 63 & 0.004 \\
GO:0005829 & Cytosol & 8 & 0.017 \\
GO:0014069 & Postsynaptic density & 48 & 0.018 \\
GO:0046872 & Metal ion binding & 4 & 0.002 \\
GO:0008373 & Sialyltransferase activity & 26 & 0.003 \\
GO:0003700 & Nranscription factor activity, sequence-specific DNA binding & 26 & 0.005 \\
GO:0003676 & Nucleic acid binding & 152 & 0.007 \\
GO:0005515 & Protein binding & 11 & 0.008 \\
hsa05202 & Transcriptional misregulation in cancer & 16 & $5.20-04$ \\
hsa05200 & Pathways in cancer & 5 & 0.002 \\
hsa04146 & Peroxisome & 0.048 \\
\hline
\end{tabular}

GO: Gene Ontology; KEGG: Kyoto Encyclopedia of Genes and Genomes; DEGs: differentially expressed genes. COAD: colon adenocarcinoma.

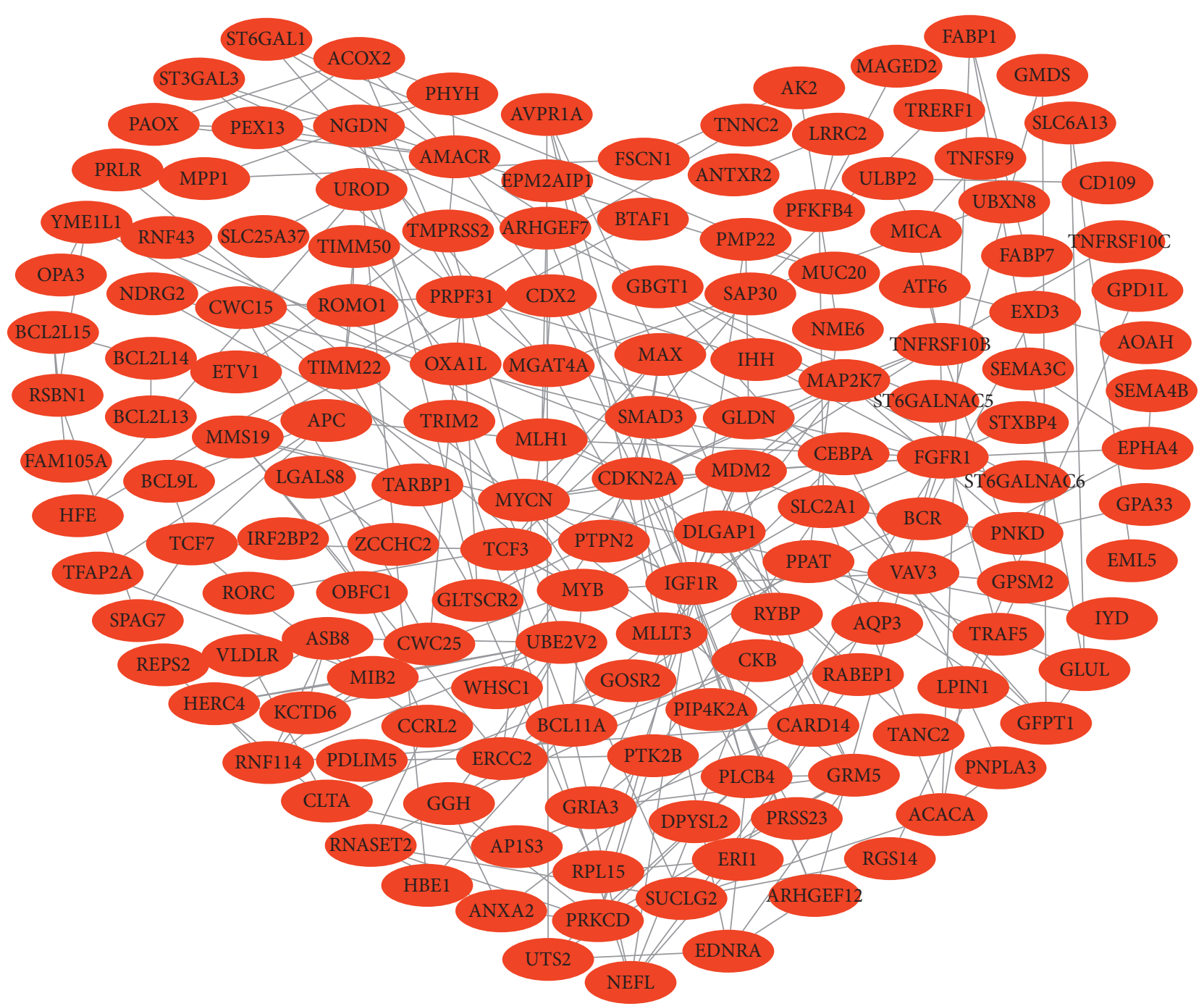

FIGURE 2: Protein-protein interaction network of differentially expressed genes, consisting of 264 edges and 159 nodes. 
CDKN2A, IGF1R, MDM2, SMAD3, SLC2A1, GRM5, PLCB4, FGFR1, UBE2V2, and TNFRSF10B (Figure 3). The results of $\mathrm{GO}$ analysis showed that variations in the $\mathrm{BP}$ were largely enriched in the activation of cysteine-type endopeptidase activity involved in the apoptotic process, activation of cysteine-type endopeptidase activity involved in the apoptotic signaling pathway, protein destabilization, protein K63-linked ubiquitination, and immune response. Variations in the CC were predominantly enriched in receptor complex, integral component of plasma membrane, plasma membrane, and cytosol, whereas those in the MF were enriched in identical protein binding, SUMO transferase activity, ubiquitin protein ligase binding, protein binding, and p53 binding. KEGG pathway analysis revealed that the hub genes were mainly enriched in pathways in cancer, adherens junction, cell cycle, FoxO signaling pathway, and proteoglycans in cancer (Table 3). Summaries of the functions of all hub genes are presented in Table 4.

3.5. Analysis of Hub Genes. A coexpression network of the hub genes was constructed using cBioPortal. Among these genes, CDKN2A, UBE2V2, MDM2, SMAD3, FGFR1, IGF1R, and PLCB4 exhibited the highest node scores, suggesting that they may possess pivotal functions for distinguishing between LCOAD and RCOAD (Figure 4). Using the BiNGO tool, biological process analysis of the hub genes is illustrated in Figure 5(a), and molecular function analyses of the hub genes are presented in Figure 5(b). Hierarchical clustering revealed that the hub genes were able to differentiate between the LCOAD and RCOAD samples (Figure 6). Within the GSE81558 dataset, when compared with LCOAD, the expression of GRM5 and UBE2V2 was downregulated, and that of CDKN2A, SLC2A1, IGF1R, FGFR1, TNFRSF10B, MDM2, SMAD3, and PLCB4 was upregulated in RCOAD (Figure 6(a)). In the GSE75317 dataset, when compared with LCOAD, expression levels of PLCB4 and UBE2V2 were downregulated, while those of CDKN2A, MDM2, TNFRSF10B, SMAD3, and SLC2A1 were upregulated in RCOAD (Figure 6(b)).

3.6. RT-qPCR Analysis Validation of Hub Genes. As presented in the result, GRM5 and PLCB4 were markedly downregulated in RCOAD samples, when compared with the LCOAD. The relative expression levels of CDKN2A, IGF1R, MDM2, SMAD3, SLC2A1, FGFR1, UBE2V2, and TNFRSF10B were significantly higher in RCOAD samples, compared with the LCOAD groups (Figure 7). It should be noted that CDKN2A, MDM2, SMAD3, SLC2A1, and TNFRSF10B were consistent with the above results.

3.7. The Relationship between Pathological Stage, OS, and the Expression of Hub Genes. GEPIA analysis showed that the expression of CDKN2A, MDM2, SLC2A1, and TNFRSF10B was significantly associated with pathological stage $(P<0.05$; Figures $8(\mathrm{a}), 8(\mathrm{c}), 8(\mathrm{~d})$, and $8(\mathrm{e}))$, while the expression of IGF1R, SMAD3, GRM5, PLCB4, FGFR1, and UBE2V2 was not (Figures $8(\mathrm{~b}), 8(\mathrm{~d}), 8(\mathrm{f})$ and $9(\mathrm{a})-9(\mathrm{c}))$. The pathological stage of COAD was positively related to the expression of CDKN2A and SLC2A1 and negatively related to the expression of MDM2 and TNFRSF10B. Kaplan-Meier analysis using GEPIA revealed that COAD patients with high expression levels of CDKN2A had poorer overall survival times than those with low expression levels $(P<0.05$; Figure 10(a)); there was no statistically significant effect on OS associated with the expression of IGF1R, MDM2, SMAD3, SLC2A1, GRM5, PLCB4, FGFR1, UBE2V2, or TNFRSF10B $(P>0.05$; Figures 10(b)-10(i)). Therefore, the other nine genes are not related to the prognosis. After the analysis by GEPIA, there exists a positive correlation between SLC2A1 and GLUT1 expression levels $(R=1$, $P<0.001)$.

3.8. High Expressions of CDKN2A in Patients with LCOAD or RCOAD Were Independent Prognostic Factors for the Poor Overall Survival. The demographic data and the expression status of CDKN2A were summarized in Table 5. The Kaplan-Meier OS curves were presented in Figure 11. High expression of CDKN2A was a predictor of a shorter OS in the LCOAD patients (Figure 11(a)) and RCOAD patients (Figure 11(b)).

\section{Discussion}

With global changes in diet and lifestyle, COAD-associated morbidity and mortality have increased, making it one of the primary malignant tumors threatening human health. There is no consensus on the relationship between tumor location and the pathological stage and prognosis of COAD. A metaanalysis [30] of 66 studies that analyzed the OS data of 1.43 million COAD patients showed a $19 \%$ reduction in mortality among patients with LCOAD, compared with those with RCOAD; this suggested that the location of the primary tumor serves a key role in determining the prognosis of colon adenocarcinoma. However, Weiss et al. [7] found no significant difference in the 5-year OS rates between patients with left and right COAD, following the adjustment for various prognostic factors. In addition, numerous studies have reported differences in the molecular mechanisms of COAD at different locations $[10,31,32]$, but it was not clear whether these molecular differences could be translated into clinically meaningful changes in pathological stage and prognosis. Therefore, pathological stage and prognosis may serve important roles in investigating the relationship between the molecular mechanisms of the occurrence and development of COAD at different locations, facilitating the screening, diagnosis, and targeted treatment of patients with COAD [33].

Bioinformatics is the computational science of understanding biological and genetic information for the purpose of expanding the use of biological and medical data [34]. The units of bioinformatics research are DNA, RNA, and protein molecules, which can be reliably utilized for the identification and investigation of DEGs $[35,36]$. COAD results from the interaction of multiple genes and the bioinformatic application of gene expression profiles provide the 


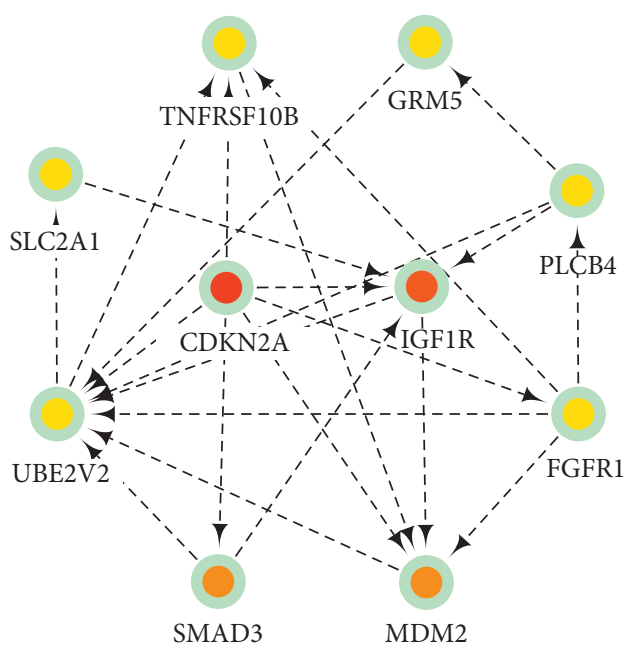

FIGURE 3: Hub genes identified within the protein-protein interaction network.

TABLE 3: GO and KEGG pathway enrichment analyses of hub genes between left and right COAD.

\begin{tabular}{|c|c|c|c|}
\hline Term & Description & Count in gene set & $P$ value \\
\hline GO:0006919 & Activation of cysteine-type endopeptidase activity involved in apoptotic process & 3 & $8.50 E-04$ \\
\hline GO:0097296 & Activation of cysteine-type endopeptidase activity involved in apoptotic signaling pathway & 2 & 0.007 \\
\hline GO:0031648 & Protein destabilization & 2 & 0.019 \\
\hline GO:0070534 & Protein K63-linked ubiquitination & 2 & 0.020 \\
\hline GO:0006955 & Immune response & 3 & 0.020 \\
\hline GO:0043235 & Receptor complex & 3 & 0.002 \\
\hline GO:0005887 & Integral component of plasma membrane & 5 & 0.003 \\
\hline GO:0005886 & Plasma membrane & 7 & 0.006 \\
\hline GO:0005829 & Cytosol & 6 & 0.013 \\
\hline GO:0042802 & Identical protein binding & 5 & $4.05 E-04$ \\
\hline GO:0019789 & SUMO transferase activity & 2 & 0.009 \\
\hline GO:0031625 & Ubiquitin protein ligase binding & 3 & 0.010 \\
\hline GO:0005515 & Protein binding & 9 & 0.026 \\
\hline GO:d0002039 & p53 binding & 2 & 0.035 \\
\hline hsa05200 & Pathways in cancer & 7 & $8.51 E-07$ \\
\hline hsa04520 & Adherens junction & 3 & 0.003 \\
\hline hsa04110 & Cell cycle & 3 & 0.008 \\
\hline hsa04068 & FoxO signaling pathway & 3 & 0.010 \\
\hline hsa05205 & Proteoglycans in cancer & 3 & 0.021 \\
\hline
\end{tabular}

GO: Gene Ontology; KEGG: Kyoto Encyclopaedia of Genes and Genomes; DEGs: differentially expressed genes; COAD: colon adenocarcinoma.

possibility of studying the pathogenesis of COAD at different locations. Furthermore, the biological analysis of gene chip data is another important advancement for data mining [37].

In the present study, bioinformatics technology was used to analyze two datasets (GSE81558 and GSE75317), in which a total of 286 DEGs were identified. GO enrichment analysis, KEGG signal pathway analysis, and PPI network analysis were also performed with these DEGs, and the following ten hub genes associated with COAD at different locations were identified by the cytoHubba when the degree $\geq 10$, one plugin of Cytoscape software: CDKN2A, IGF1R, MDM2, SMAD3, SLC2A1, GRM5, PLCB4, FGFR1, UBE2V2, and TNFRSF10B. Among these genes, the expression of CDKN2A and SLC2A1 was upregulated in RCOAD, compared with LCOAD. GEPIA showed that the expression of CDKN2A was significantly associated with pathological stage $(P<0.05)$. With the increase in CDKN2A expression levels, the pathological stage of COAD also increased $(P<0.05)$. Kaplan-Meier curve analysis using GEPIA revealed that $\mathrm{COAD}$ patients with high expression levels of CDKN2A had poorer OS times than those with low expression levels $(P<0.05)$.

Cyclin-Dependent Kinase Inhibitor 2A (CDKN2A) is an important tumor suppressor gene belonging to the family of cyclin-dependent kinase inhibitor genes, which serves a regulatory role in cell proliferation and apoptosis [38]. The pathways associated with CDKN2A are signaling and apoptosis modulation. CDKN2A codes for two cyclic inhibitory proteins, p16INK4a and p14ARF. Furthermore, through the p16ink4a-cdk4 (and CDK6)-prb and p14arfmdm2-p53 pathways, it serves a role in cell cycle regulation. CDKN2A is able to induce cell cycle arrest at the $G_{1}$ and $G_{2}$ phases and thus has a tumor-inhibitory effect [39]. CDKN2A 
TABLE 4: Summaries for the function of 10 hub genes.

\begin{tabular}{|c|c|c|c|}
\hline No. & Gene symbol & Full name & Function \\
\hline 1 & CDKN2A & $\begin{array}{c}\text { Cyclin-dependent kinase } \\
\text { inhibitor } 2 \mathrm{~A}\end{array}$ & $\begin{array}{l}\text { Capable of inducing cell cycle arrest in G1 and G2 phases. Acts as a tumor suppressor. } \\
\text { Acts as a negative regulator of the proliferation of normal cells by interacting strongly } \\
\text { with CDK4 and CDK6 }\end{array}$ \\
\hline 2 & IGF1R & $\begin{array}{l}\text { Insulin-like growth factor } 1 \\
\text { receptor }\end{array}$ & $\begin{array}{l}\text { The activated IGF1R is involved in cell growth and survival control. IGF1R is crucial for } \\
\text { tumor transformation and survival of malignant cell }\end{array}$ \\
\hline 3 & MDM2 & MDM2 proto-oncogene & $\begin{array}{l}\text { Inhibits p53/TP53- and p73/TP73-mediated cell cycle arrest and apoptosis by binding } \\
\text { its transcriptional activation domain. Inhibits DAXX-mediated apoptosis by inducing } \\
\text { its ubiquitination and degradation }\end{array}$ \\
\hline 4 & SMAD3 & SMAD family member 3 & $\begin{array}{l}\text { Receptor-regulated SMAD (R-SMAD) that is an intracellular signal transducer and } \\
\text { transcriptional modulator activated by TGF-beta (transforming growth factor) and } \\
\text { activin type } 1 \text { receptor kinases }\end{array}$ \\
\hline 5 & SLC2A1 & $\begin{array}{l}\text { Solute carrier family } 2 \\
\text { member } 1\end{array}$ & $\begin{array}{l}\text { Facilitative glucose transporter. This isoform may be responsible for constitutive or } \\
\text { basal glucose uptake, has a very broad substrate specificity, and can transport a wide } \\
\text { range of aldoses including both pentoses and hexoses }\end{array}$ \\
\hline 6 & GRM5 & $\begin{array}{l}\text { Glutamate metabotropic } \\
\text { receptor } 5\end{array}$ & $\begin{array}{c}\text { Ligand binding causes a conformation change that triggers signaling via guanine } \\
\text { nucleotide-binding proteins ( } \mathrm{G} \text { proteins) and modulates the activity of down-stream } \\
\text { effectors }\end{array}$ \\
\hline 7 & PLCB4 & Phospholipase C beta 4 & $\begin{array}{c}\text { The production of the second messenger molecules diacylglycerol (DAG) and inositol } \\
\text { 1,4,5-trisphosphate (IP3) is mediated by activated phosphatidylinositol-specific } \\
\text { phospholipase C enzymes }\end{array}$ \\
\hline 8 & FGFR1 & $\begin{array}{l}\text { Fibroblast growth factor } \\
\text { receptor } 1\end{array}$ & $\begin{array}{c}\text { Tyrosine-protein kinase that acts as cell-surface receptor for fibroblast growth factors } \\
\text { and plays an essential role in the regulation of embryonic development, cell } \\
\text { proliferation, differentiation, and migration }\end{array}$ \\
\hline 9 & UBE2V2 & $\begin{array}{l}\text { Ubiquitin conjugating } \\
\text { enzyme E2 V2 }\end{array}$ & $\begin{array}{l}\text { Plays a role in the control of progress through the cell cycle and differentiation, plays a } \\
\text { role in the error-free DNA repair pathway, and contributes to the survival of cells after } \\
\text { DNA damage }\end{array}$ \\
\hline 10 & TNFRSF10B & $\begin{array}{l}\text { TNF receptor superfamily } \\
\text { member } 10 \mathrm{~b}\end{array}$ & Promotes the activation of NF-kappa-B. Essential for ER stress-induced apoptosis \\
\hline
\end{tabular}

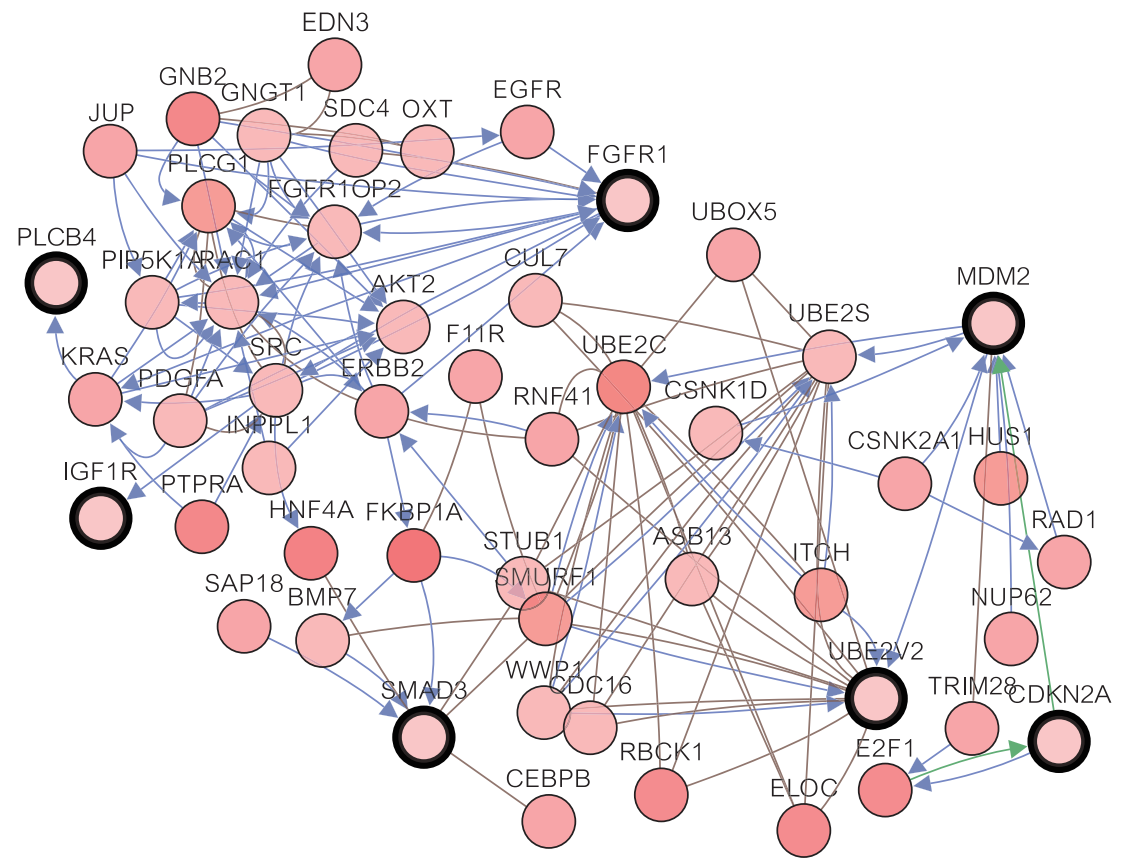

Figure 4: Coexpression network of hub genes obtained using cBioPortal.

binds the proto-oncogene MDM2 and blocks its karyoplasmic shuttling by sequestrating MDM2 in the nucleolus. In addition, MDM2-induced degradation of p53 was blocked, enhancing p53-dependent activation and subsequent apoptosis, thereby inhibiting the carcinogenic effect of MDM2 [40]. Additionally, CDKN2A is able to bind BCL6, downregulating bcl6-induced transcriptional inhibition; it can also bind $\mathrm{E} 2 \mathrm{~F} 1$ and $\mathrm{MYC}$, blocking the 


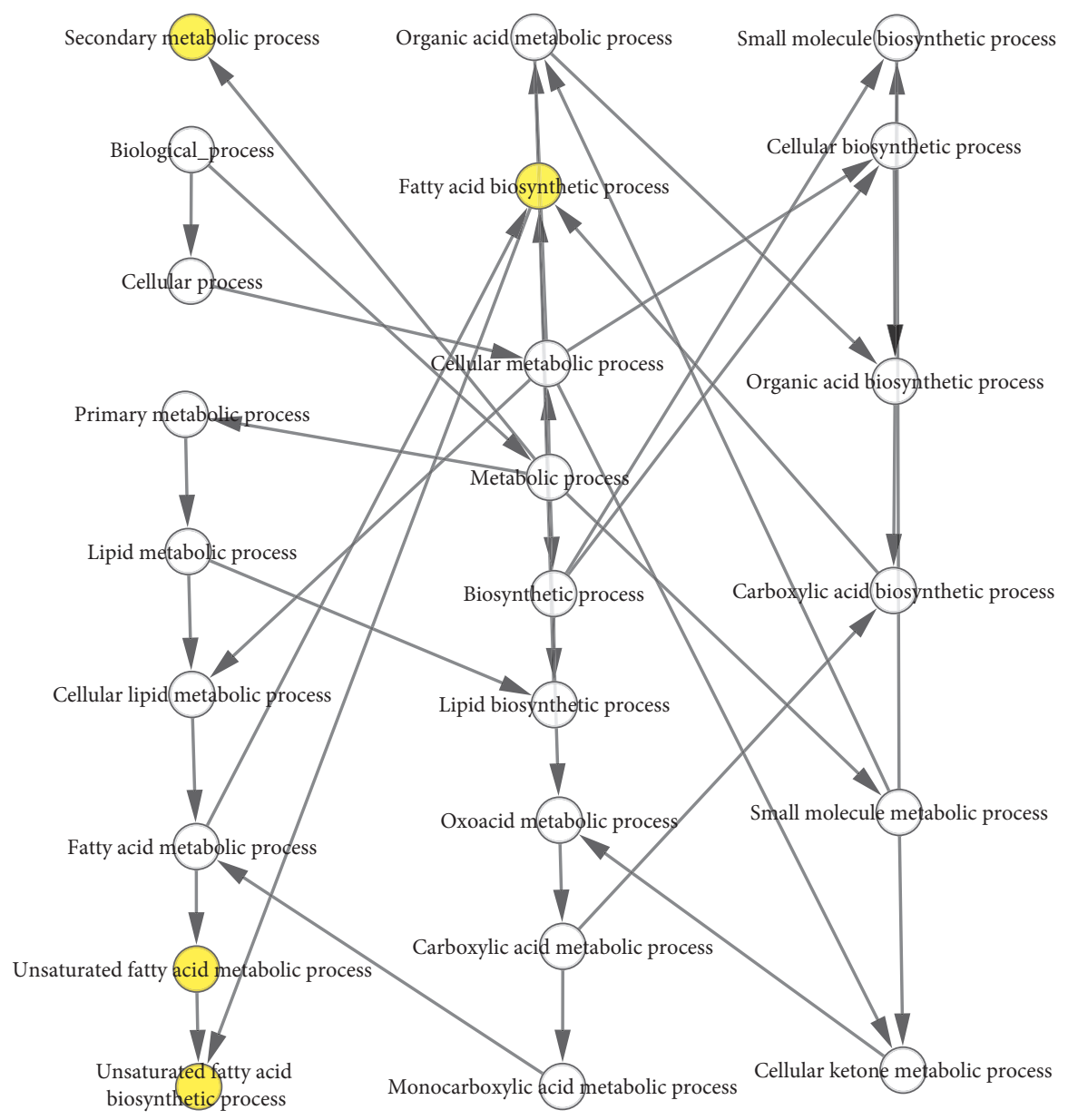

(a)

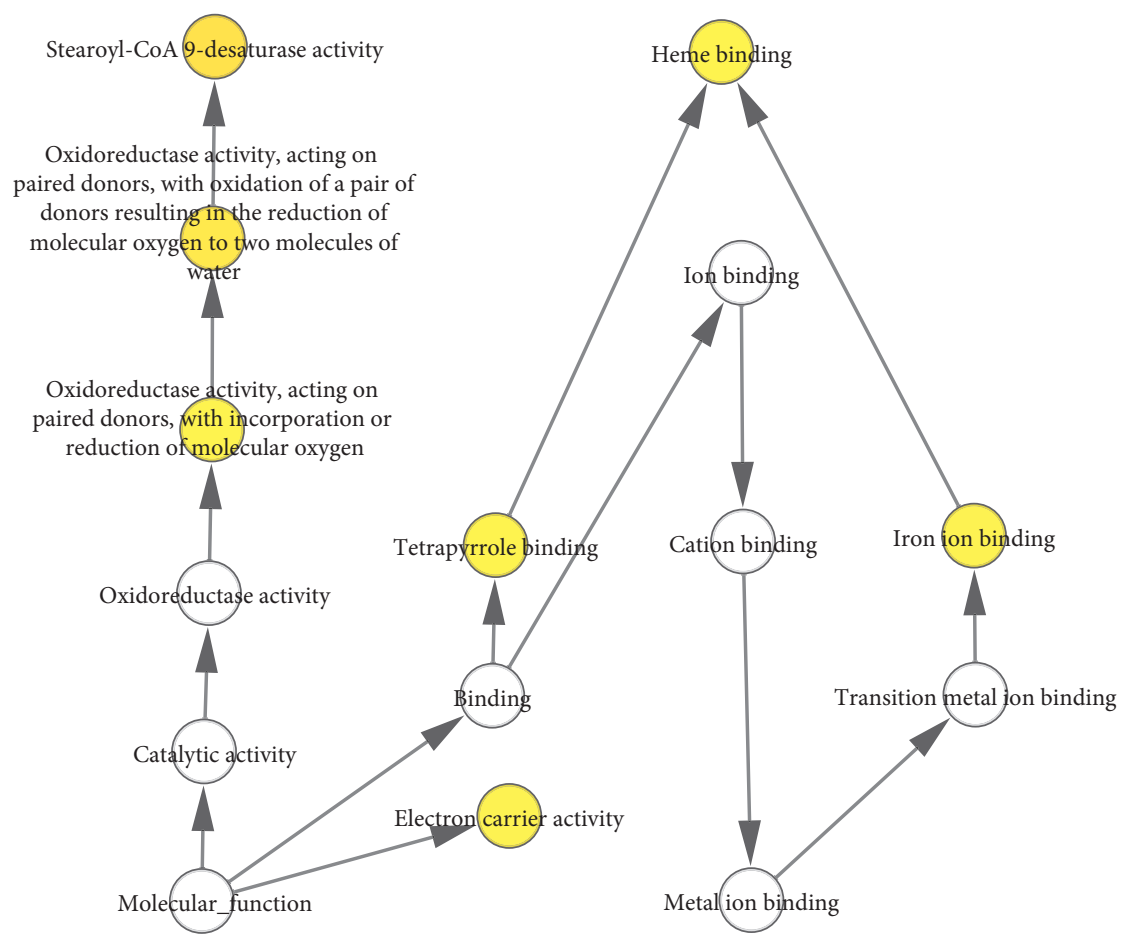

(b)

Figure 5: (a) Biological process and (b) molecular function analysis of the identified hub genes using the Biological Networks Gene Oncology tool. 

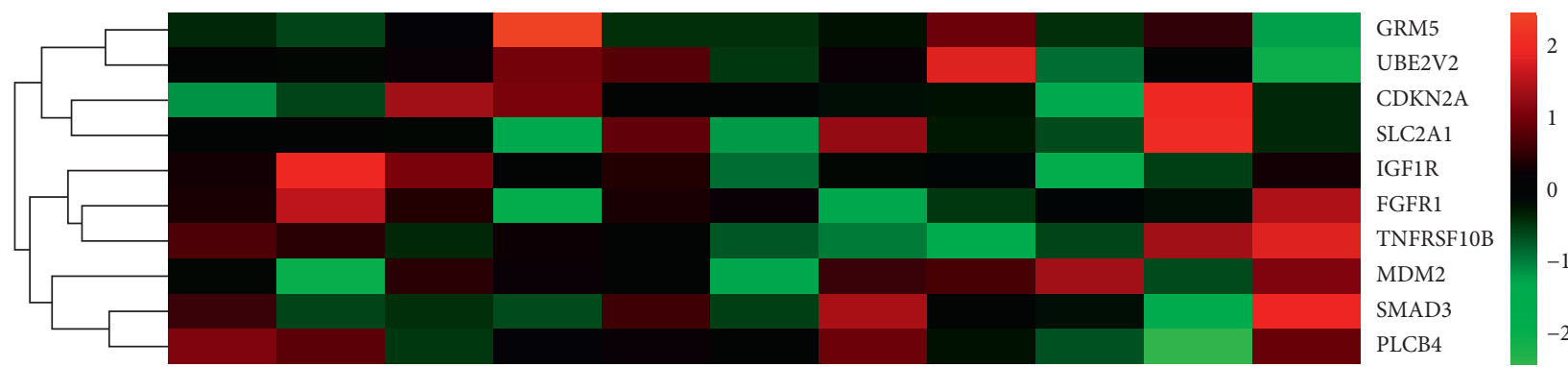

\begin{tabular}{|c|c|c|c|c|c|c|c|c|}
\hline 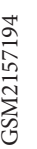 & 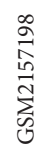 & 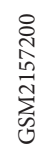 & 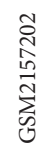 & 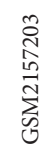 & 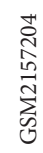 & 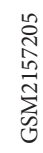 & 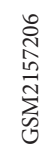 & \\
\hline
\end{tabular}

(a)

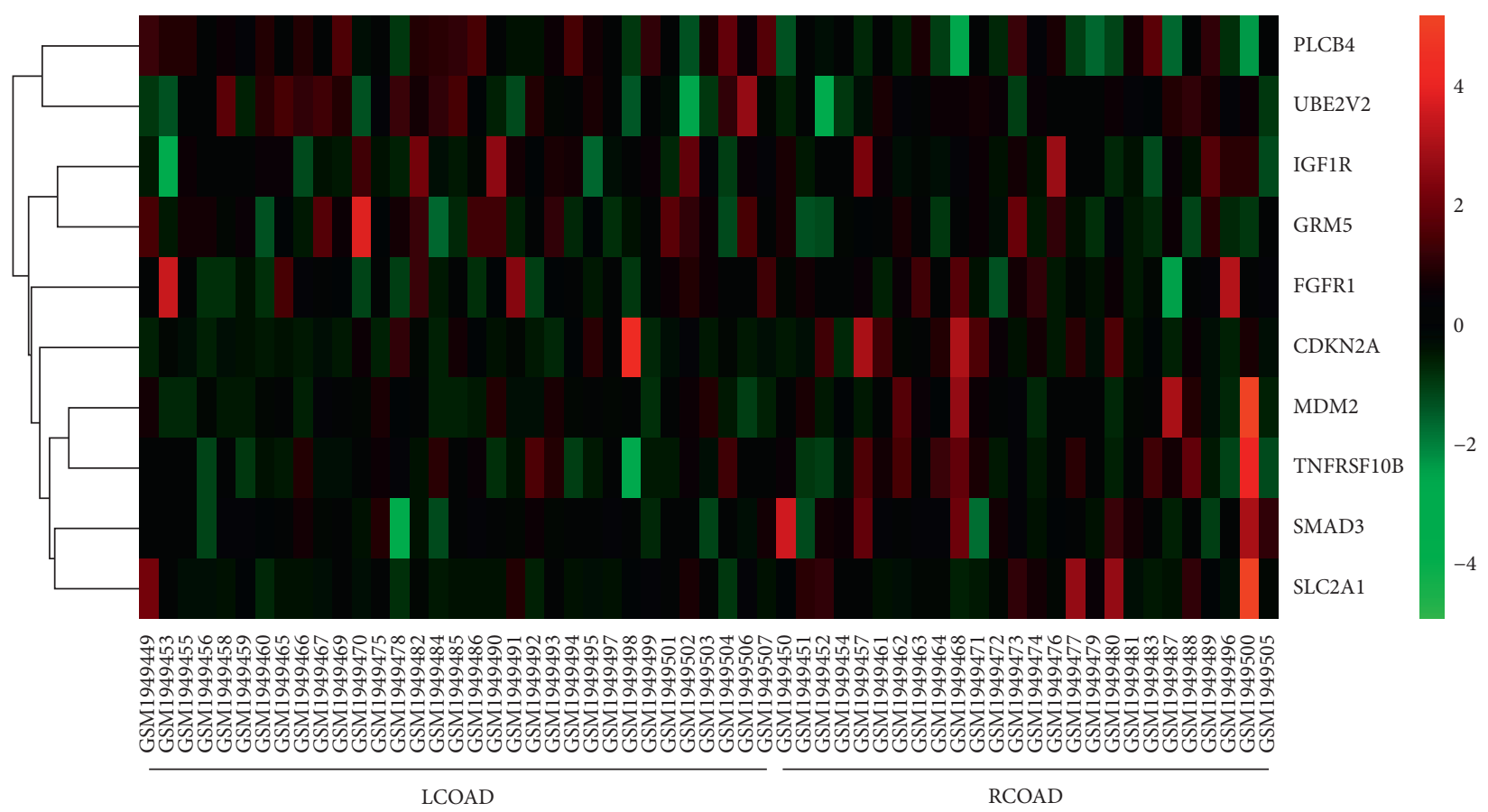

(b)

FIGURE 6: Hierarchical clustering. Differentiation between RCOAD and LCOAD samples in the (a) GSE81558 and (b) GSE75317 datasets using the identified hub genes. The color represents the expression level of each gene (green, low expression; black, medium expression; and red, high expression).

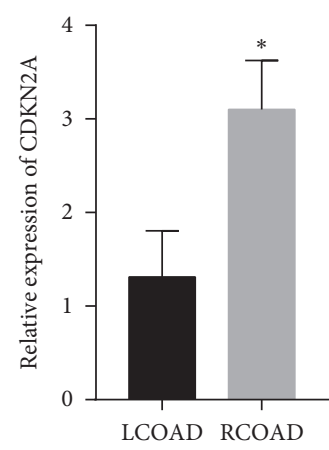

(a)

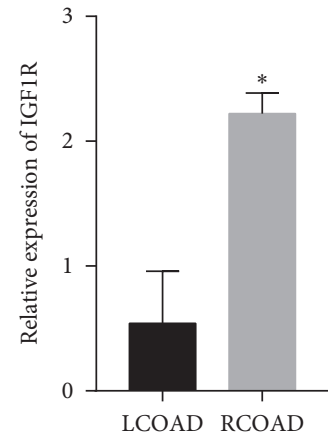

(b)

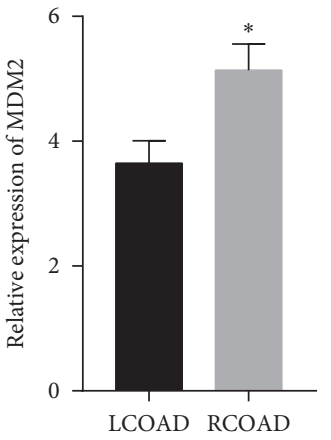

(c)

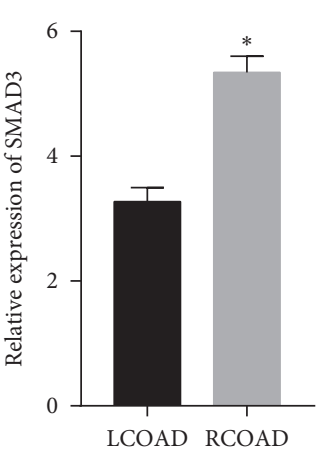

(d)

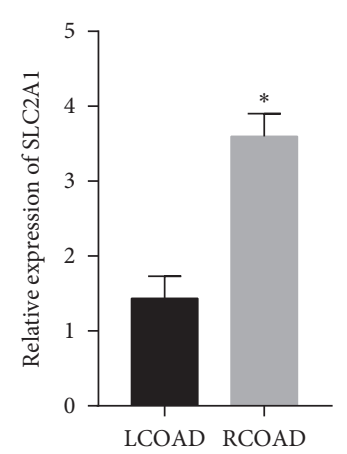

(e)

Figure 7: Continued. 


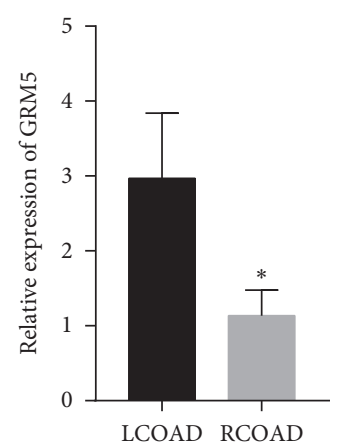

(f)

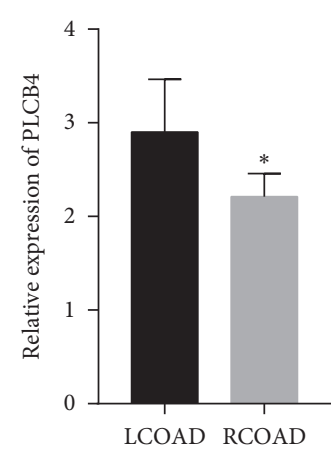

(g)

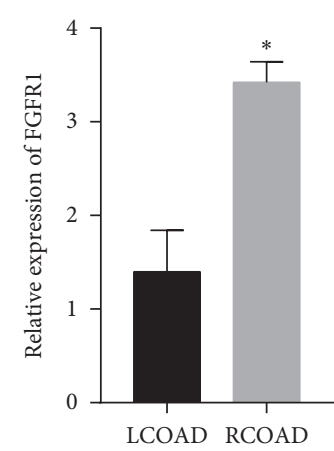

(h)

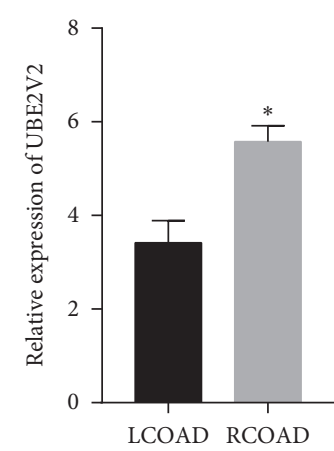

(i)

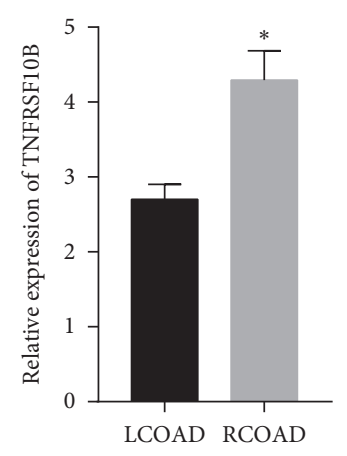

(j)

FIGURE 7: Relative expression of hub genes between LCOAD and RCOAD by RT-qPCR analysis. (a) CDKN2A, (b) IGF1R, (c) MDM2, (d) SMAD3, (e) SLC2A1, (f) GRM5, (g) PLCB4, (h) FGFR1, (i) UBE2V2, and (j) TNFRSF10B. ${ }^{*} P<0.05$.

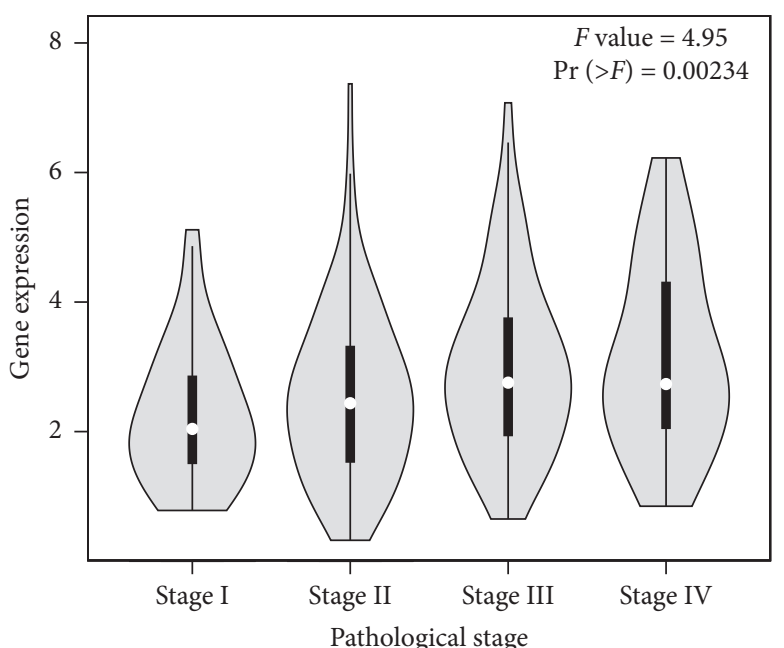

(a)

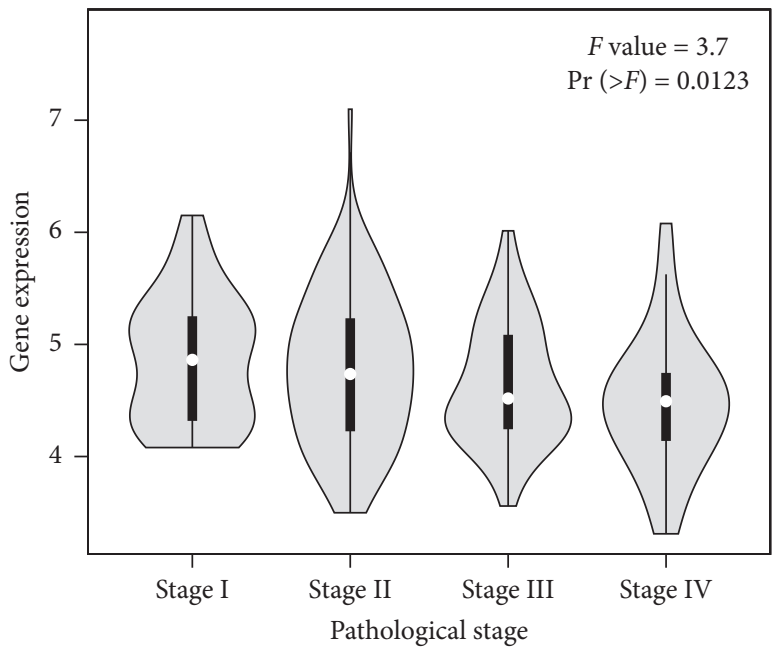

(c)

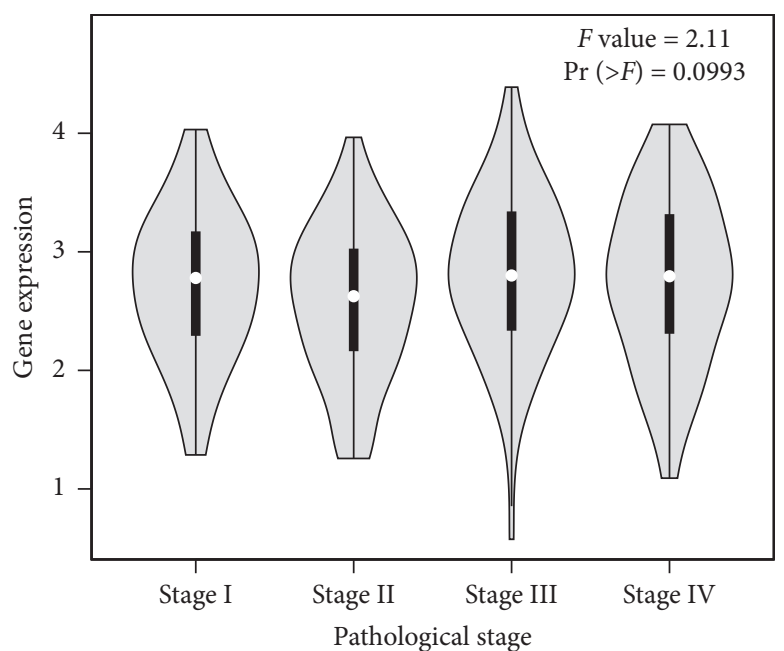

(b)

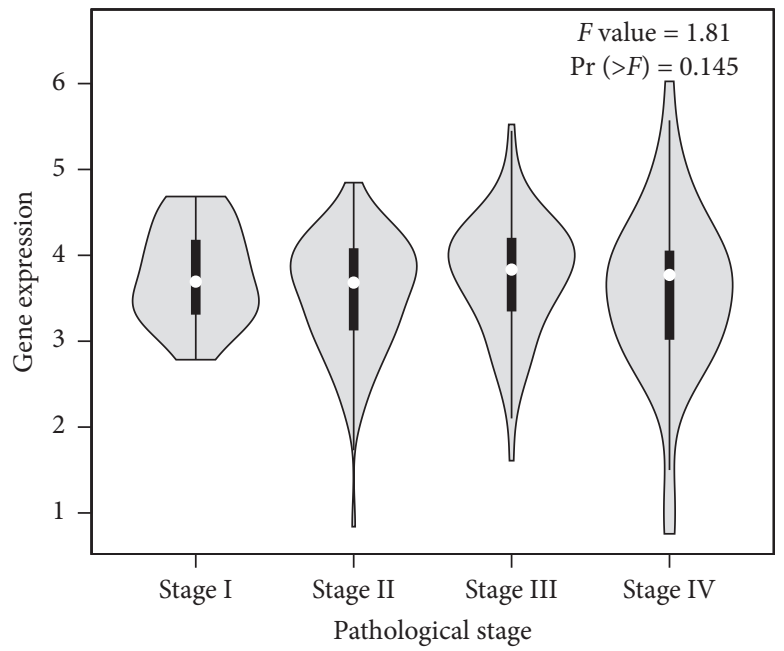

(d)

FIgURE 8: Continued. 


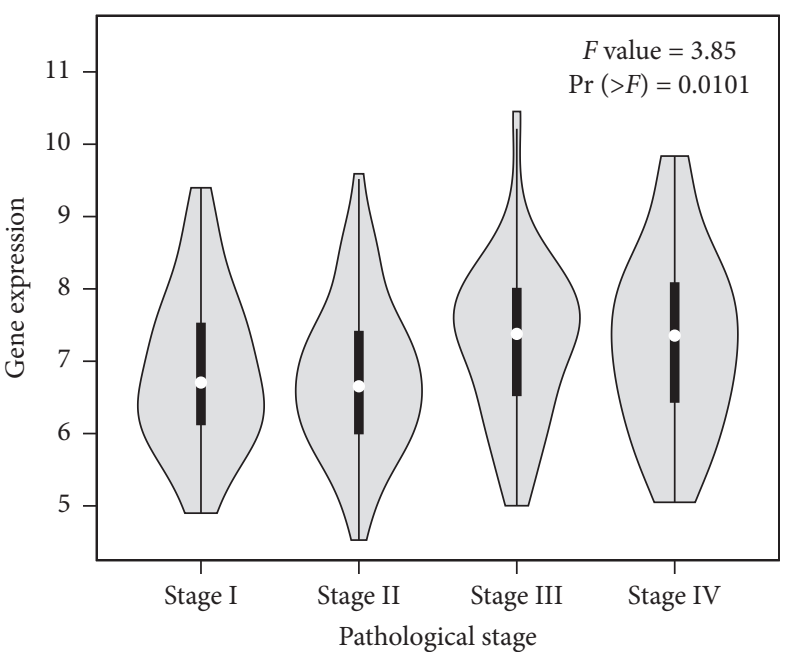

(e)

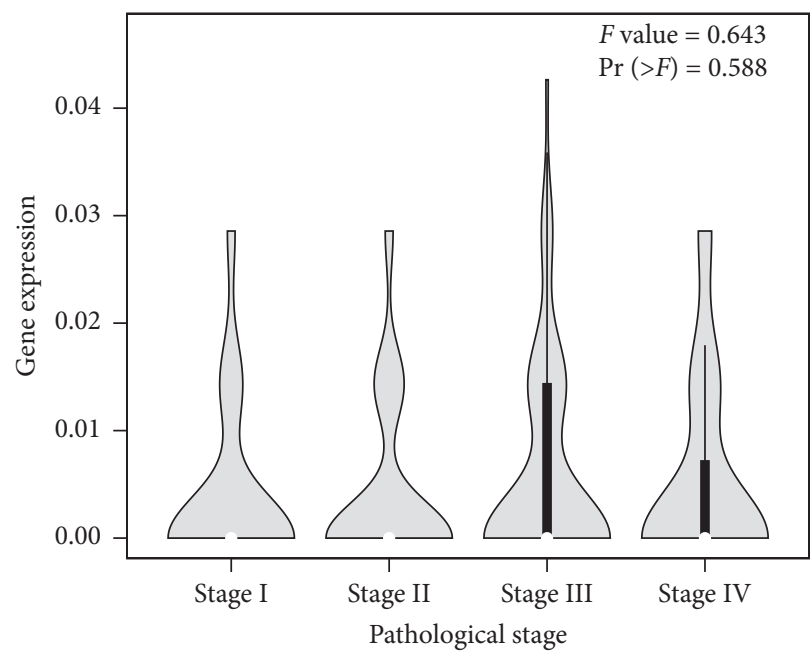

(f)

FIGURE 8: Association between pathological stage and the expression levels of (a) CDKN2A, (b) IGF1R, (c) MDM2, (d) SMAD3, (e) SLC2A1, and (f) GRM5.

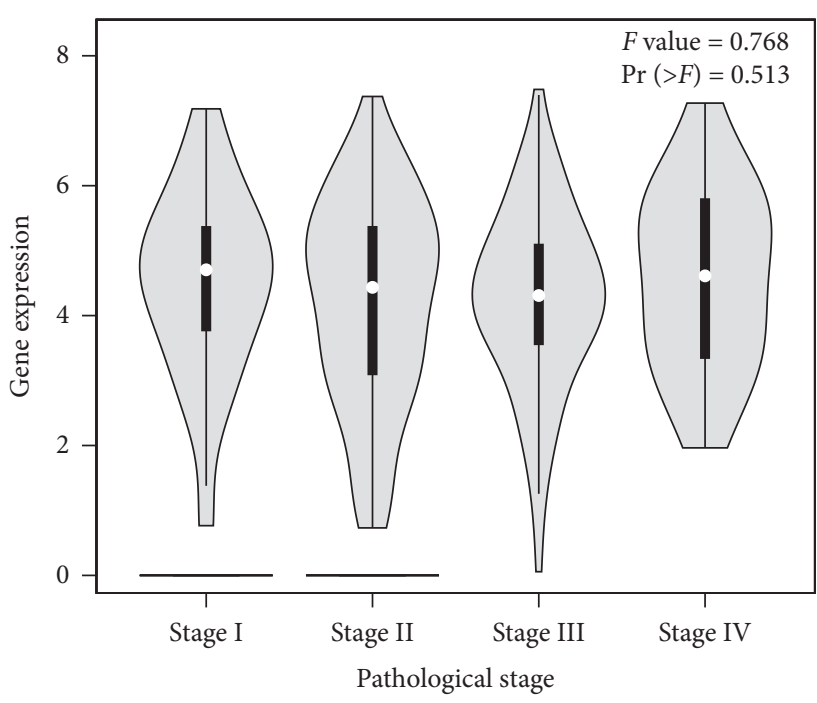

(a)

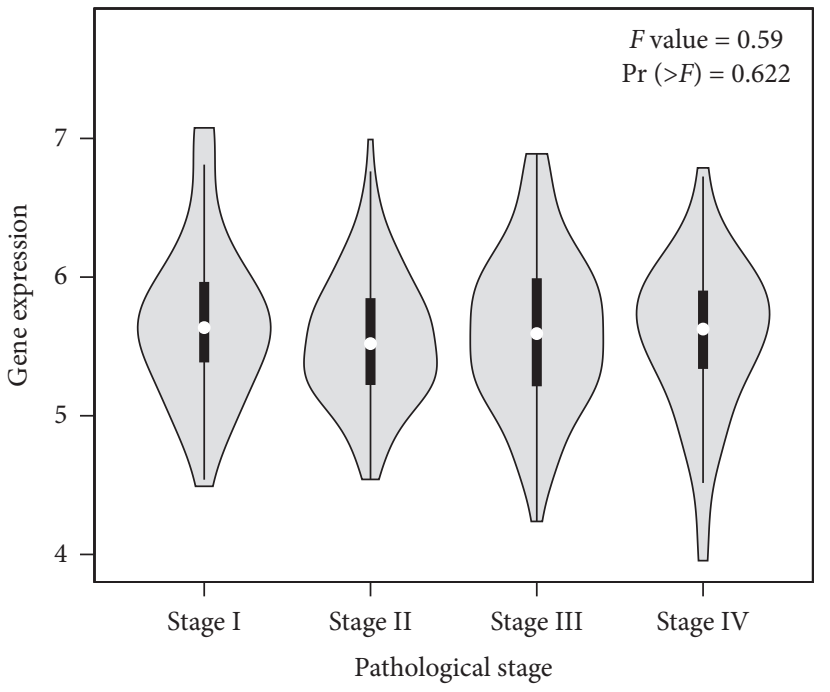

(c)

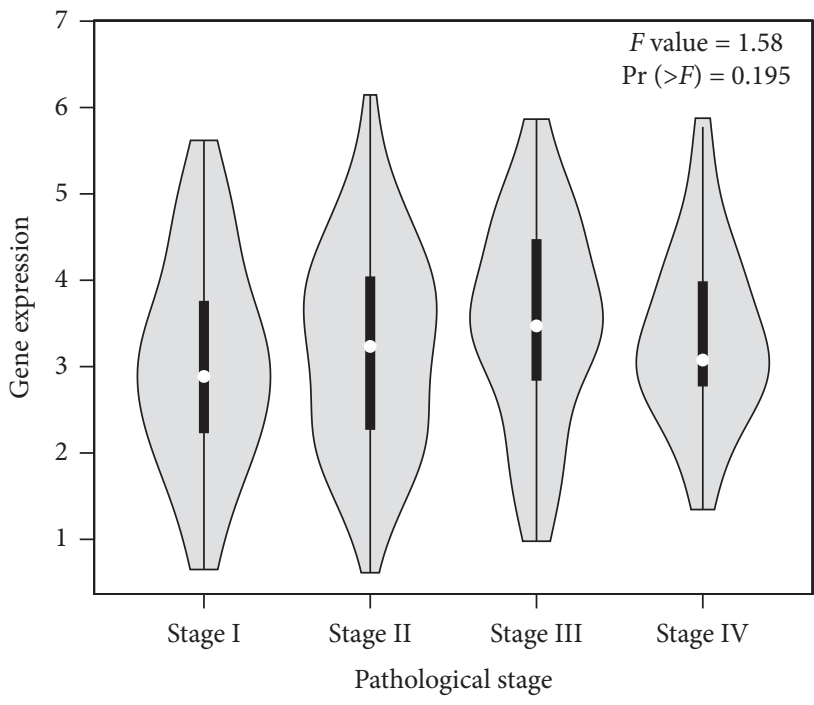

(b)

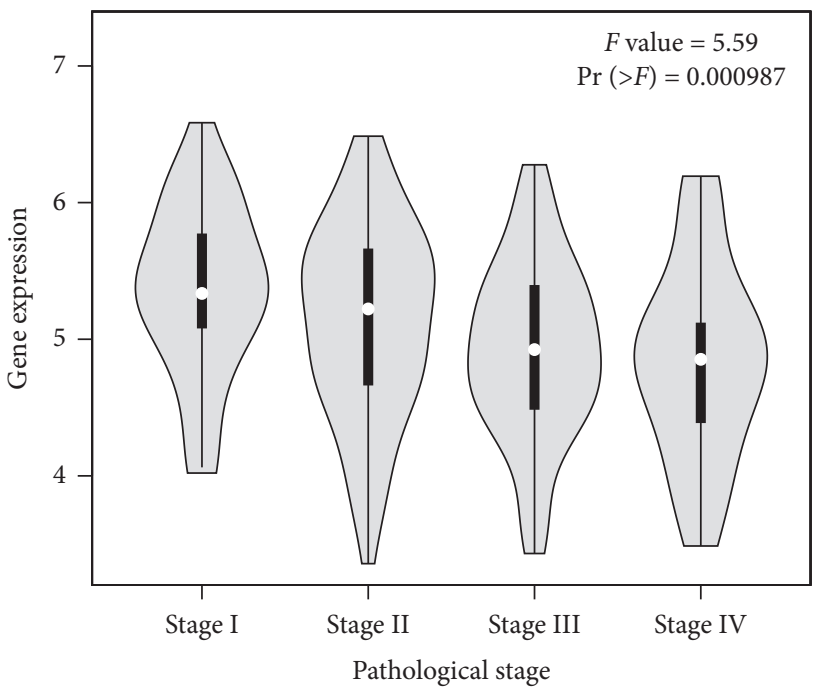

(d)

FIgURE 9: Association between pathological stage and the expression levels of (a) PLCB4, (b) FGFR1, (c) UBE2V2, and (d) TNFRSF10B. 


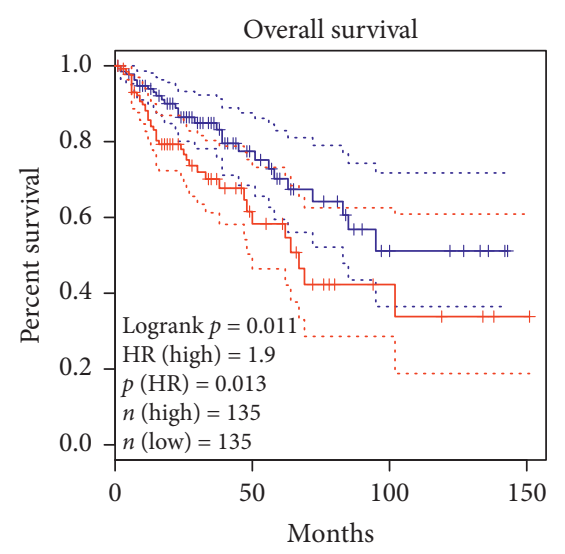

— Low CDKN2A TPM
High CDKN2A TPM

(a)

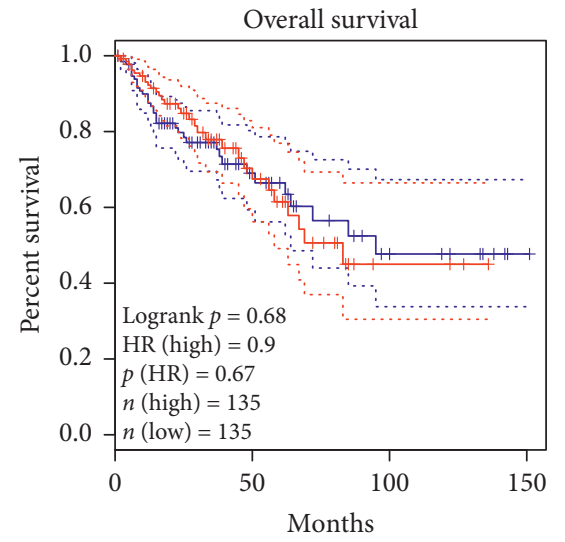

— Low SMAD3 TPM

- High SMAD3 TPM

(d)

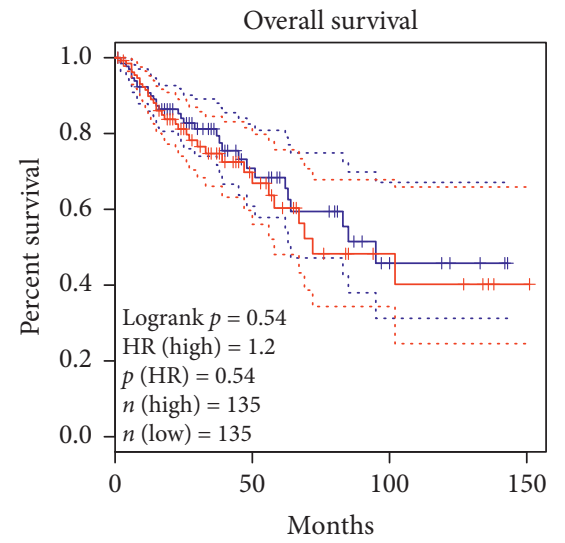

— Low FGFR1 TPM

- High FGFR1 TPM

(g)

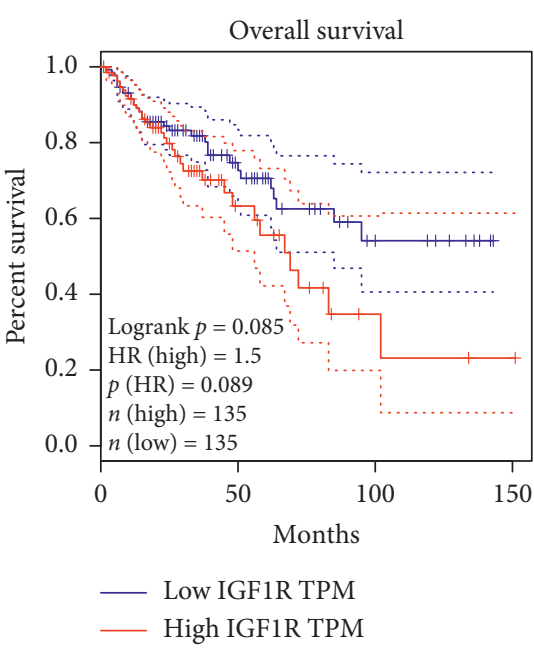

(b)

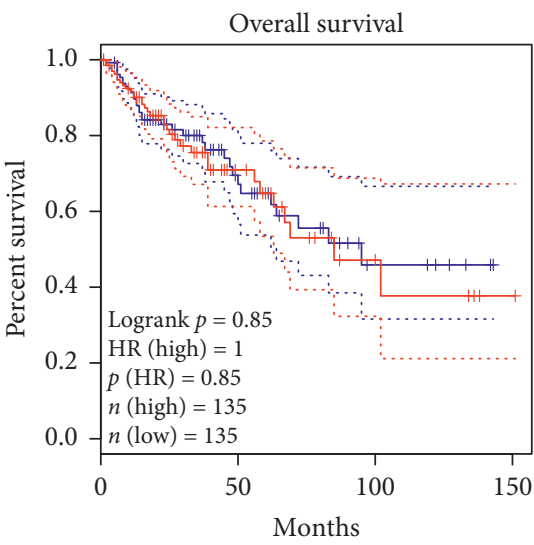

_ Low SLC2A1 TPM

- High SLC2A1 TPM

(e)

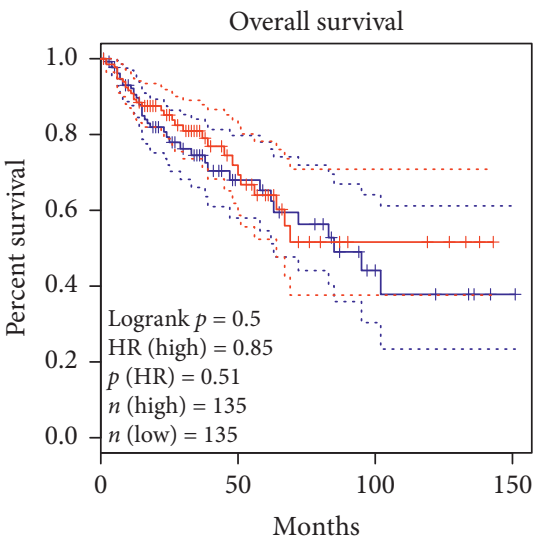

— Low UBE2V2 TPM

— High UBE2V2 TPM

(h)

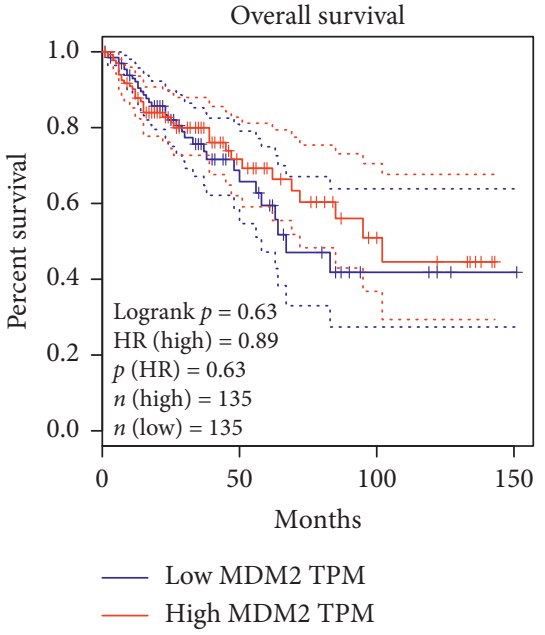

(c)

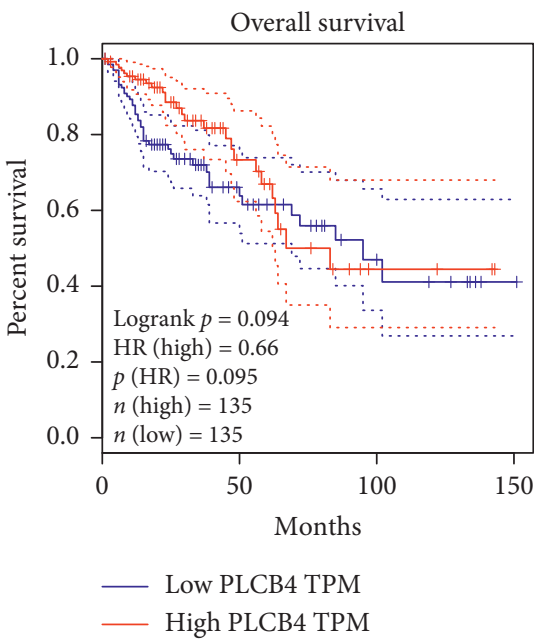

(f)

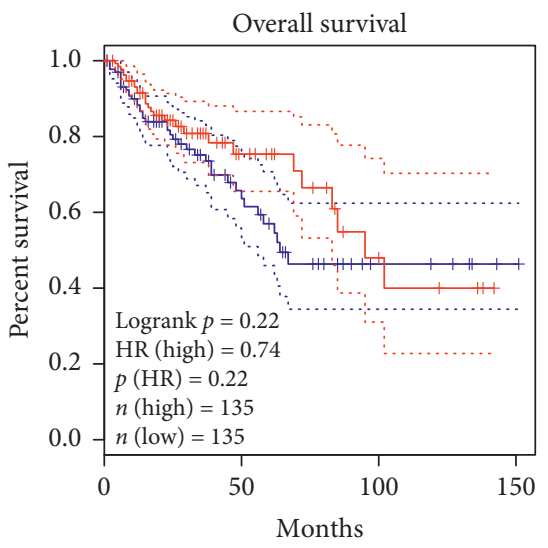

— Low TNFRSF10B TPM

— High TNFRSF10B TPM

(i)

FIGURE 10: Kaplan-Meier overall survival analysis using Gene Expression Profiling Interactive Analysis. (a) CDKN2A, (b) IGF1R, (c) MDM2, (d) SMAD3, (e) SLC2A1, (f) PLCB4, (g) FGFR1, (h) UBE2V2, and (i) TNFRSF10B. The expression level of CDKN2A is closely correlated with the prognosis of COAD patients $(P<0.05)$. 
TABLE 5: The demographic data and the expression status of CDKN2A.

\begin{tabular}{lcccc}
\hline & & & & CDKN2A \\
& & & Low (\%) & High (\%) \\
\hline \multirow{2}{*}{ Sex } & Male & 181 & $119(56.1 \%)$ & $02(29.2 \%)$ \\
& Female & 31 & $0(0.0 \%)$ & $31(14.6 \%)$ \\
\hline \multirow{2}{*}{ Age } & $<65$ years & 100 & $64(30.2 \%)$ & $36(17.0 \%)$ \\
& $\geq 65$ years & 112 & $55(25.9 \%)$ & $57(26.9 \%)$ \\
\multirow{2}{*}{ Tumor location } & LCOAD & 106 & $59(27.8 \%)$ & $47(22.2 \%)$ \\
& RCOAD & 106 & $60(28.3 \%)$ & $46(21.7 \%)$ \\
\hline \multirow{2}{*}{ Overall survival } & $<60$ months & 122 & $57(26.9 \%)$ & $65(30.7 \%)$ \\
& $\geq 60$ months & 90 & $62(29.2 \%)$ & $28(13.2 \%)$ \\
\hline
\end{tabular}

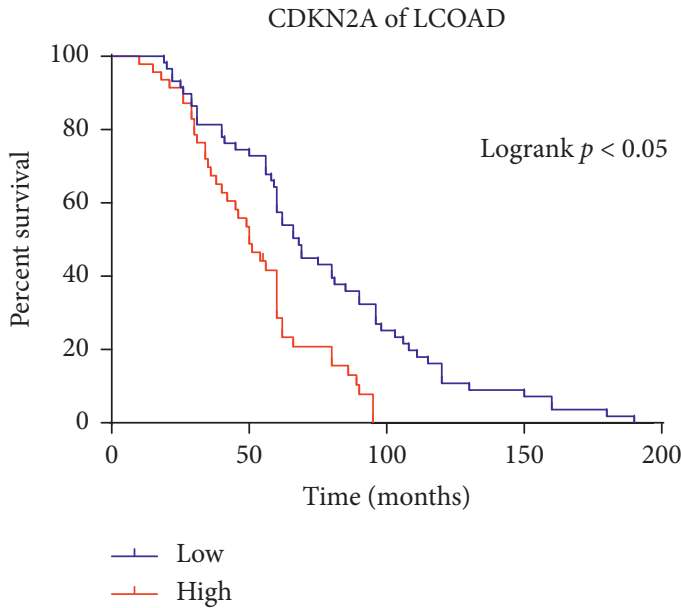

(a)

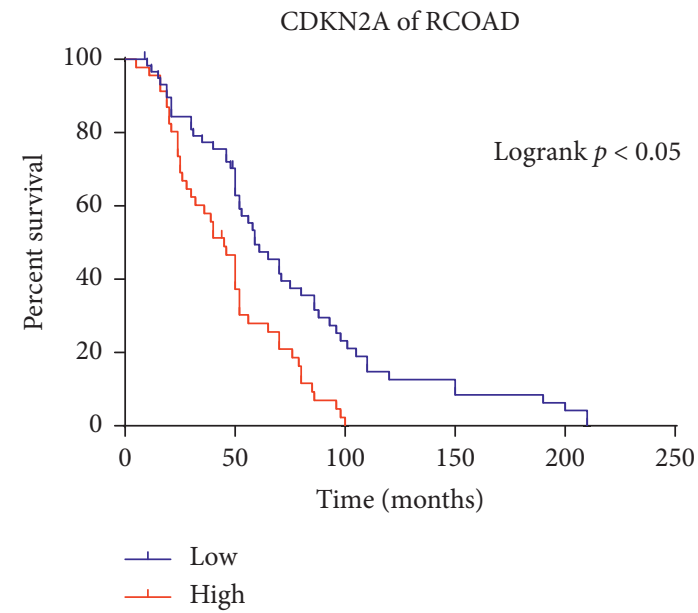

(b)

Figure 11: The Kaplan-Meier OS curves of the LCOAD and RCOAD patients with the low/high expression of CDKN2A. (a) High expression of CDKN2A was a predictor of a shorter OS in the LCOAD patients $(P<0.05)$. (b) High expression of CDKN2A was a predictor of a shorter OS in the RCOAD patients $(P<0.05)$.

transcriptional activation activity of E2F1. However, no effect on MYC-associated transcriptional inhibition has been reported.

CDKN2A mutation has been demonstrated as an important event in a number of tumor types, including pancreatic cancer [41] and gastric cancer. Therefore, the development of cancer is often accompanied by CDKN2A mutations; the loss of its anticancer function may promote the neoplastic transformation of cells, subsequently inducing proliferation, invasion, and metastasis [42]. In the present study, it was speculated that CDKN2A may be mutated in $\mathrm{COAD}$, the pathological stage of COAD was positively related to the expression of CDKN2A, and the mutated protein may promote the abnormal proliferation and differentiation of colonic glandular epithelial cells. The results indicated that the expression level of CDKN2A in RCOAD was higher than that in LCOAD and that this is positively correlated with the pathological stage of patients with COAD. Survival analysis also revealed that when CDKN2A was highly expressed, the OS rate of patients with COAD was low and the prognosis was poor. This suggested a possible reason (and research direction) for the hypothesis that, at the molecular level, patients with RCOAD possess a higher pathological stage and poorer prognosis than those with LCOAD.

However, there are still some shortcomings to the present study. The sample size of only two datasets was relatively small. In the result of hierarchical clustering data, PLCB4 expression was upregulated in RCOAD as compared to LCOAD using the GSE81558 dataset while PLCB4 expression was downregulated using the GSE75317 dataset. We think that the reasons causing this situation are small sample sizes and individual differences. Currently, there are some research studies about the difference between RCOAD and LCOAD in genomics. Based on the previous studies, our study creatively identified critical differentially expressed genes between LCOAD and RCOAD through the bioinformatics method and further verified them in clinical samples. We found that CDKN2A is expected to be a key target for the pathogenesis and treatment of LCOAD and RCOAD. Meanwhile, a large number of clinical samples and animal experiments would provide more comprehensive verification and a deeper understanding of the different molecular mechanisms, clinical pathological staging, and survival differences between RCOAD and LCOAD. 


\section{Conclusion}

We studied the gene difference between LCOAD and RCOAD by bioinformatics and verified the result by molecular biology, in an attempt to deeply understand the pathogenesis of COAD and expand the thinking for the discovery of new therapeutic targets. Our study identified 286 differentially expressed genes and 10 hub genes, with a focus on verifying the differential expression and prognostic value of CDKN2A. The expression of CDKN2A is upregulated in the RCOAD and is downregulated in the LCOAD. The higher the expression of CDKN2A is, the poorer the pathological stage and overall survival are. Therefore, the prognosis of LCOAD is better than RCOAD. The present study has provided a reference point for the in-depth study of COAD-associated genes, the discovery of molecular markers at different locations, and the biological processes in which they are involved.

$\begin{array}{ll}\text { Abbreviations } \\ \text { COAD: } & \text { Colon adenocarcinoma } \\ \text { LCOAD: } & \text { Left colon adenocarcinoma } \\ \text { RCOAD: } & \text { Right colon adenocarcinoma } \\ \text { GEO: } & \text { Gene expression omnibus } \\ \text { DEGs: } & \text { Differentially expressed genes } \\ \text { GO: } & \text { Gene Ontology } \\ \text { KEGG: } & \text { Kyoto Encyclopedia of Genes and Genomes } \\ \text { PPI: } & \text { Protein-protein interaction } \\ \text { GEPIA: } & \text { Gene expression profiling interactive analysis } \\ \text { BP: } & \text { Biological process } \\ \text { CC: } & \text { Cellular component } \\ \text { MF: } & \text { Molecular function } \\ \text { BiNGO: } & \text { Biological networks gene oncology tool } \\ \text { OS: } & \text { Overall survival } \\ \text { CDKN2A: } & \text { Cyclin-dependent kinase inhibitor 2A } \\ \text { MDM2: } & \text { MDM2 proto-oncogene } \\ \text { SLC2A1: } & \text { Solute carrier family } 2 \text { member 1. }\end{array}$

\section{Data Availability}

The datasets used and/or analyzed during the current study are available from the corresponding author on reasonable request.

\section{Conflicts of Interest}

The authors declare that they have no conflicts of interest.

\section{Authors' Contributions}

$\mathrm{JH}$ performed the experiments and was a major contributor in writing and submitting the manuscript. GYW made substantial contributions to the research conception, and XZ designed the draft of the research proposal. YY was involved in critically revising the manuscript for important intellectual content, and data analysis was performed by LF. All authors read and approved the final manuscript.

\section{Acknowledgments}

The authors are thankful to the Department of Medical Oncology, the Fourth Hospital of Hebei Medical University, for the assistance and support during the submission process.

\section{References}

[1] L. A. Torre, F. Bray, R. L. Siegel, J. Ferlay, J. Lortet-Tieulent, and A. Jemal, "Global cancer statistics, 2012," CA: A Cancer Journal for Clinicians, vol. 65, no. 2, pp. 87-108, 2015.

[2] D. K. Rex, D. J. Ahnen, J. A. Baron et al., "Serrated lesions of the colorectum: review and recommendations from an expert panel," American Journal of Gastroenterology, vol. 107, no. 9, pp. 1315-1329, 2012.

[3] K. Tamas, A. M. E. Walenkamp, E. G. E. de Vries et al., "Rectal and colon cancer: not just a different anatomic site," Cancer Treatment Reviews, vol. 41, no. 8, pp. 671-679, 2015.

[4] L. Liang, J.-H. Zeng, X.-G. Qin, J.-Q. Chen, D.-Z. Luo, and G. Chen, "Distinguishable prognostic signatures of left- and right-sided colon cancer: a study based on sequencing data," Cellular Physiology and Biochemistry, vol. 48, no. 2, pp. 475-490, 2018.

[5] H. S. Saidi, D. Karuri, and E. O. Nyaim, "Correlation of clinical data, anatomical site and disease stage in colorectal cancer," East African Medical Journal, vol. 85, no. 6, pp. 259-262, 2008.

[6] T. Nawa, J. Kato, H. Kawamoto et al., "Differences between right- and left-sided colon cancer in patient characteristics, cancer morphology and histology," Journal of Gastroenterology and Hepatology, vol. 23, no. 3, pp. 418-423, 2008.

[7] J. M. Weiss, P. R. Pfau, E. S. O’Connor et al., "Mortality by stage for right- versus left-sided colon cancer: analysis of surveillance, epidemiology, and end results-medicare data," Journal of Clinical Oncology, vol. 29, no. 33, pp. 4401-4409, 2011.

[8] N. Hugen, C. J. H. van de Velde, J. H. W. de Wilt, and I. D. Nagtegaal, "Metastatic pattern in colorectal cancer is strongly influenced by histological subtype," Annals of Oncology, vol. 25, no. 3, pp. 651-657, 2014.

[9] X. Wang, Q. Wei, J. Gao et al., "Clinicopathologic features and treatment efficacy of Chinese patients with BRAF-mutated metastatic colorectal cancer: a retrospective observational study," Chinese Journal of Cancer, vol. 36, no. 1, p. 81, 2017.

[10] H. Shen, J. Yang, Q. Huang et al., "Different treatment strategies and molecular features between right-sided and leftsided colon cancers," World Journal of Gastroenterology, vol. 21, no. 21, pp. 6470-6478, 2015.

[11] X. H. Gao, G. Y. Yu, H. F. Gong et al., "Differences of protein expression profiles, KRAS and BRAF mutation, and prognosis in right-sided colon, left-sided colon and rectal cancer," Scientific Reports, vol. 7, no. 1, p. 7882, 2017.

[12] M. E. Lleonart, J. S. Garcia-Foncillas, and R. Sanchez-Prieto, "Microsatellite instability and p53 mutations in sporadic right and left colon carcinoma," Cancer, vol. 83, no. 5, pp. 889-895, 1998.

[13] P. F. Rambau, M. Odida, and H. Wabinga, "p53 expression in colorectal carcinoma in relation to histopathological features in Ugandan patients," African Health Sciences, vol. 8, no. 4, pp. 234-238, 2008.

[14] P. Paluszkiewicz, H. Berbeć, B. Pawłowska-Wakowicz, M. Cybulski, and A. Paszkowska, "p53 protein accumulation in colorectal cancer tissue has prognostic value only in left- 
sided colon tumours," Cancer Detection and Prevention, vol. 28, no. 4, pp. 252-259, 2004.

[15] M. R. Ghavam-Nasiri, E. Rezaei, K. Ghafarzadegan, M. Seilanian-Toosi, and H. Malekifard, "Expression of p53 in colorectal carcinoma: correlation with clinicopathologic features," Archives of Iranian Medicine, vol. 10, no. 1, pp. 38-42, 2007.

[16] T. Olivier, P. Chappuis, and P. Tsantoulis, "Bioinformatics: a key role in oncology," Revue Médicale Suisse, vol. 12, no. 519, pp. 978-981, 2016.

[17] X. Zhang, I.-H. Cha, and K.-Y. Kim, "Use of a combined gene expression profile in implementing a drug sensitivity predictive model for breast cancer," Cancer Research and Treatment, vol. 49, no. 1, pp. 116-128, 2017.

[18] W. D. Xi, Y. J. Liu, X. B. Sun, J. Shan, L. Yi, and T. T. Zhang, "Bioinformatics analysis of RNA-seq data revealed critical genes in colon adenocarcinoma," European Review for Medical and Pharmacological Sciences, vol. 21, no. 13, pp. 3012-3020, 2017.

[19] R. Edgar, M. Domrachev, and A. E. Lash, "Gene expression omnibus: NCBI gene expression and hybridization array data repository," Nucleic Acids Research, vol. 30, no. 1, pp. 207-210, 2002.

[20] D. Huang, B. T. Sherman, Q. Tan et al., "The DAVID gene functional classification tool: a novel biological modulecentric algorithm to functionally analyze large gene lists," Genome Biology, vol. 8, no. 9, p. R183, 2007.

[21] M. Ashburner, C. A. Ball, J. A. Blake et al., "Gene ontology: tool for the unification of biology," Nature Genetics, vol. 25, no. 1, pp. 25-29, 2000.

[22] M. Kanehisa, "The KEGG database," in Novartis Foundation Symposium, vol. 247, pp. 91-103, Wiley, Hoboken, NJ, USA, 2002.

[23] M. E. Smoot, K. Ono, J. Ruscheinski, P.-L. Wang, and T. Ideker, "Cytoscape 2.8: new features for data integration and network visualization," Bioinformatics, vol. 27, no. 3, pp. 431-432, 2011.

[24] D. Szklarczyk, A. Franceschini, S. Wyder et al., "STRING v10: protein-protein interaction networks, integrated over the tree of life," Nucleic Acids Research, vol. 43, no. D1, pp. D447D452, 2015.

[25] E. Cerami, J. Gao, U. Dogrusoz et al., "The cBio cancer genomics portal: an open platform for exploring multidimensional cancer genomics data," Cancer Discovery, vol. 2, no. 5, pp. 401-404, 2012.

[26] S. Maere, K. Heymans, and M. Kuiper, "BiNGO: a cytoscape plugin to assess overrepresentation of gene ontology categories in biological networks," Bioinformatics, vol. 21, no. 16, pp. 3448-3449, 2005.

[27] Z. Tang, C. Li, B. Kang, G. Gao, C. Li, and Z. Zhang, "GEPIA: a web server for cancer and normal gene expression profiling and interactive analyses," Nucleic Acids Research, vol. 45, no. W1, pp. W98-W102, 2017.

[28] E. Osterman and B. Glimelius, "Recurrence risk after up-todate colon cancer staging, surgery, and pathology," Diseases of the Colon \& Rectum, vol. 61, no. 9, pp. 1016-1025, 2018.

[29] H. Feng, P. Guo, J. Wang, J. Xu, C. Xie, and F. Gao, "Expression of leptin and sirtuin-1 is associated with poor prognosis in patients with osteosarcoma," Pathology-Research and Practice, vol. 212, no. 4, pp. 319-324, 2016.

[30] F. Petrelli, G. Tomasello, K. Borgonovo et al., "Prognostic survival associated with left-sided vs right-sided colon cancer," JAMA Oncology, vol. 3, no. 2, p. 211, 2017.
[31] D. C. Koestler, J. Li, J. A. Baron et al., "Distinct patterns of DNA methylation in conventional adenomas involving the right and left colon," Modern Pathology, vol. 27, no. 1, pp. 145-155, 2014.

[32] A. K. Siraj, R. Bu, S. Prabhakaran et al., "A very low incidence of BRAF mutations in middle eastern colorectal carcinoma," Molecular Cancer, vol. 13, no. 1, p. 168, 2014.

[33] K. Räsänen, E. Lehtinen, K. Nokelainen et al., "Interleukin-6 increases expression of serine protease inhibitor kazal type 1 through STAT3 in colorectal adenocarcinoma," Molecular Carcinogenesis, vol. 55, no. 12, pp. 2010-2023, 2016.

[34] G. Duck, G. Nenadic, M. Filannino, A. Brass, D. L. Robertson, and R. Stevens, "A survey of bioinformatics database and software usage through mining the literature," PLoS One, vol. 11, no. 6, Article ID e0157989, 2016.

[35] A. K. Ajay, T.-M. Kim, V. Ramirez-Gonzalez, P. J. Park, D. A. Frank, and V. S. Vaidya, "A bioinformatics approach identifies signal transducer and activator of transcription-3 and checkpoint kinase 1 as upstream regulators of kidney injury molecule-1 after kidney injury," Journal of the American Society of Nephrology, vol. 25, no. 1, pp. 105-118, 2014.

[36] N. Pecina-Slaus, A. Kafka, J. K. Gotovac et al., "Comparable genomic copy number aberrations differ across astrocytoma malignancy grades," International Journal of Molecular Sciences, vol. 20, no. 5, p. 1251, 2019.

[37] J. M. Gonzalez-Calabozo, F. J. Valverde-Albacete, and C. Pelaez-Moreno, "Interactive knowledge discovery and data mining on genomic expression data with numeric formal concept analysis," BMC Bioinformatics, vol. 17, no. 1, p. 374, 2016.

[38] S. S. Padhi, S. Roy, M. Kar et al., "Role of CDKN2A/p16 expression in the prognostication of oral squamous cell carcinoma," Oral Oncology, vol. 73, pp. 27-35, 2017.

[39] M. C. Scaini, E. Rossi, P. L. de Siqueira Torres et al., "Functional impairment of p16 (INK4A) due to CDKN2A p.Gly23Asp missense mutation," Mutation Research/Fundamental and Molecular Mechanisms of Mutagenesis, vol. 671, no. 1-2, pp. 26-32, 2009.

[40] H. H. Al-Khalaf and A. Aboussekhra, "p16 controls p53 protein expression through miR-dependent destabilization of MDM2," Molecular Cancer Research, vol. 16, no. 8, pp. 1299-1308, 2018.

[41] J. Attri, R. Srinivasan, S. Majumdar, B. D. Radotra, and J. Wig, "Alterations of tumor suppressor gene p16INK4a in pancreatic ductal carcinoma," BMC Gastroenterology, vol. 5, no. 1, p. 22, 2005 .

[42] N. A. Wijetunga, T. J. Belbin, R. D. Burk et al., "Novel epigenetic changes in CDKN2A are associated with progression of cervical intraepithelial neoplasia," Gynecologic Oncology, vol. 142, no. 3, pp. 566-573, 2016. 


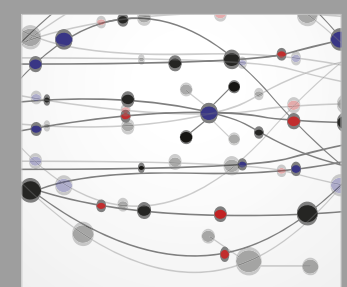

The Scientific World Journal
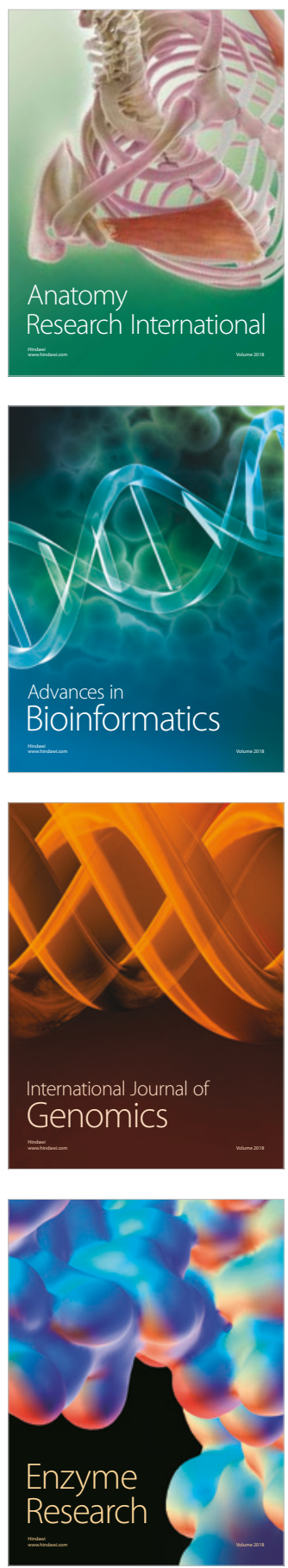
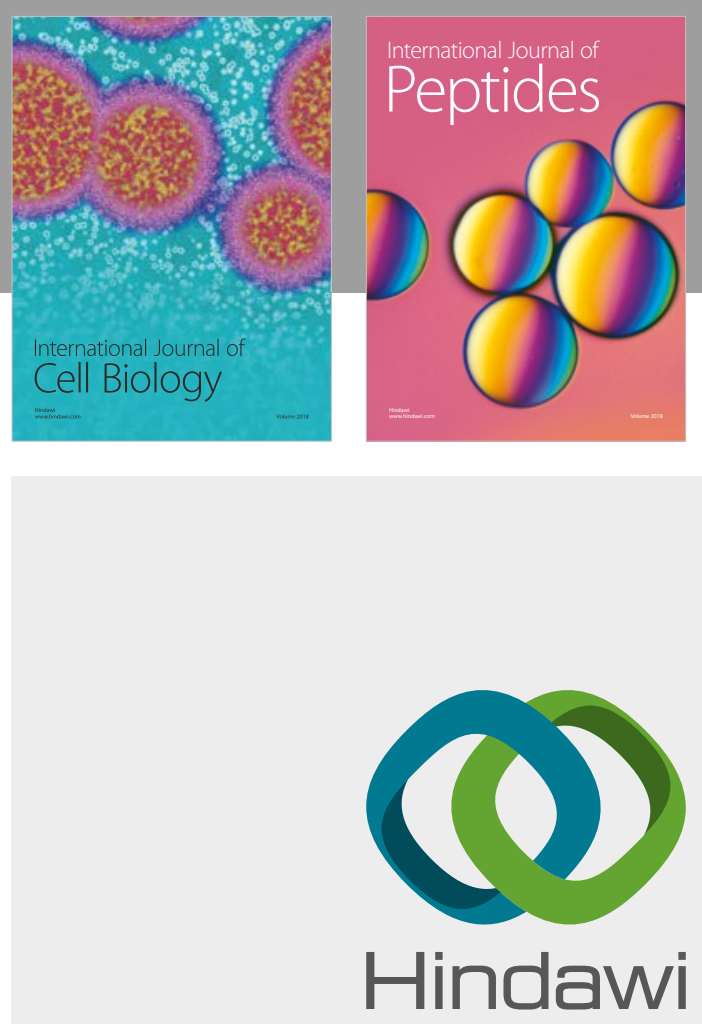

Submit your manuscripts at

www.hindawi.com
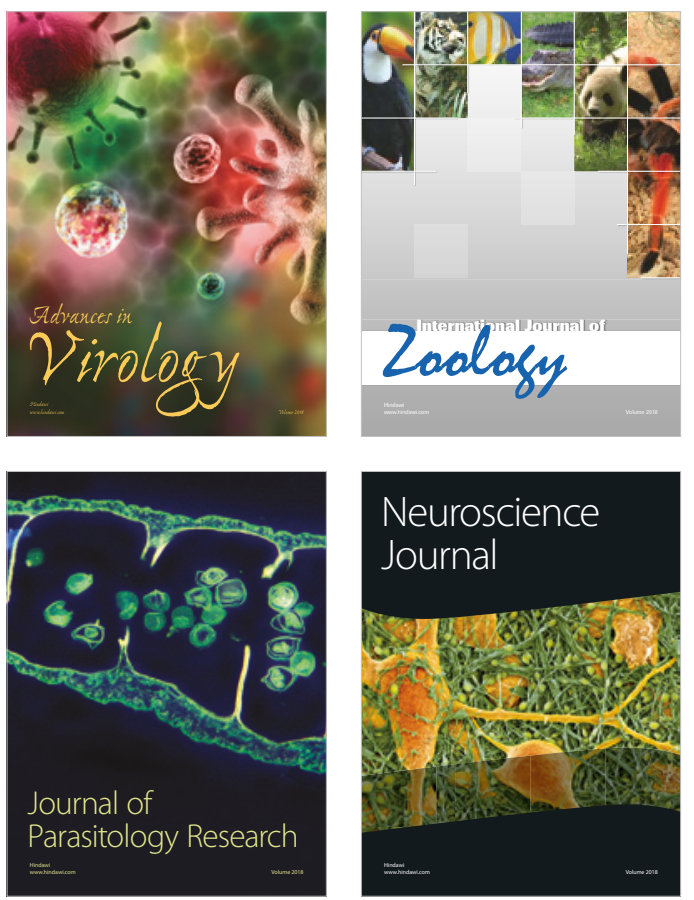
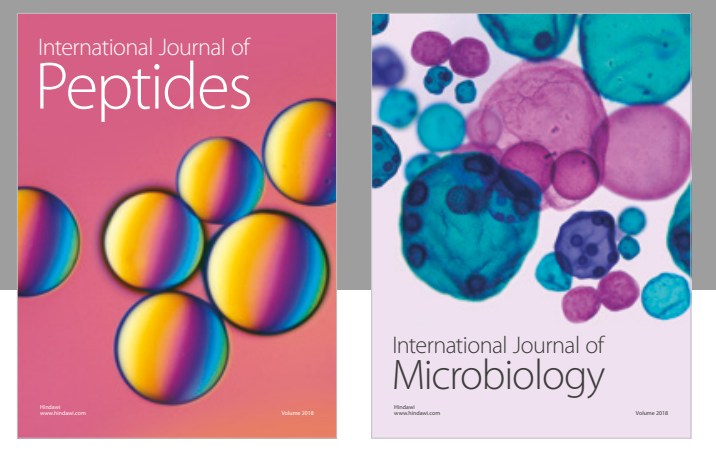

nternational Journal of Microbiology
Journal of
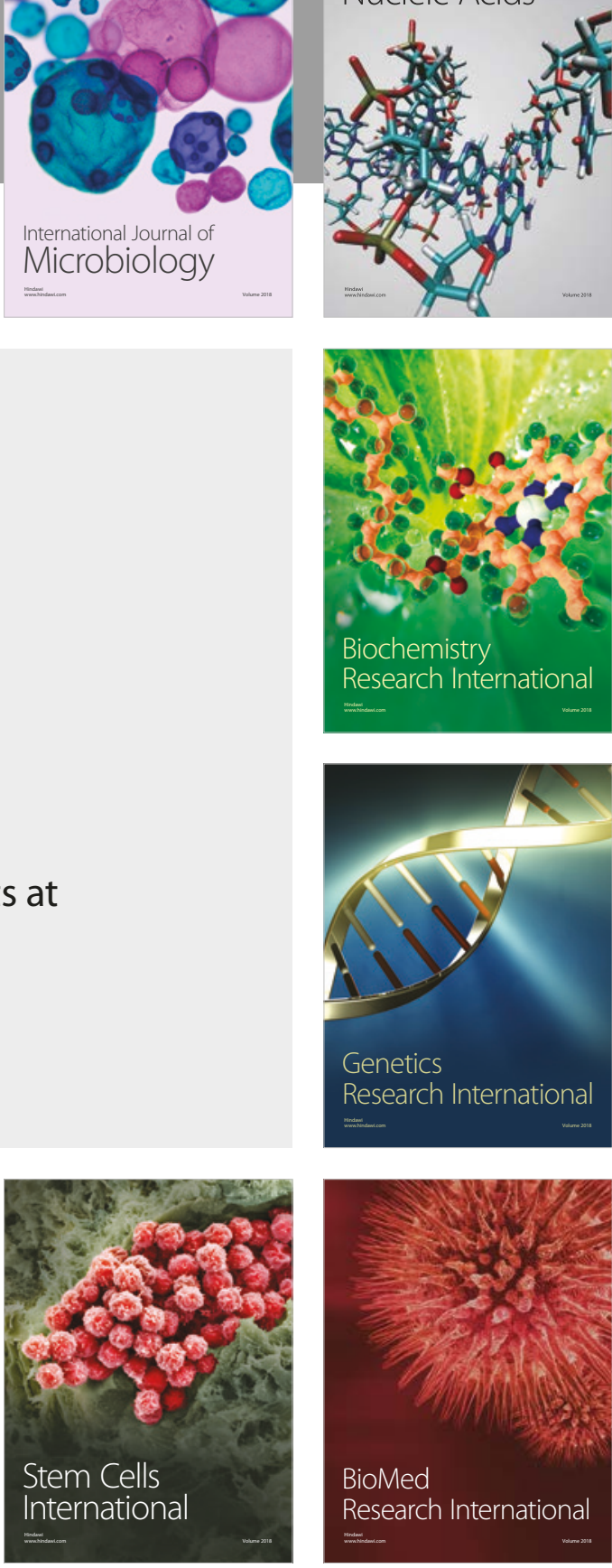
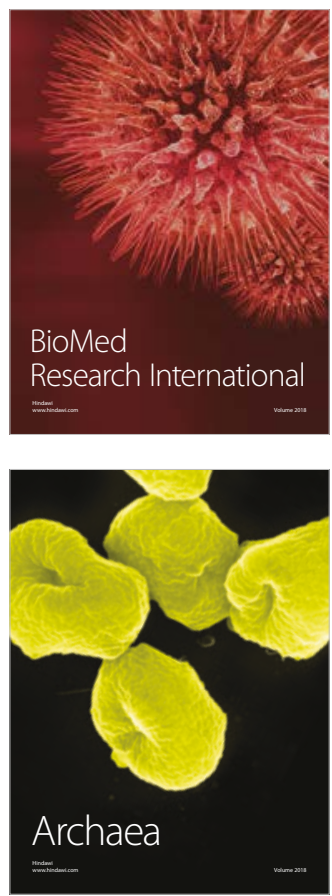